\title{
Theory of Second Order Numerical Simulation Method of Enhanced Oil Production
}

\author{
Yirang Yuan ${ }^{1}$, Aijie Cheng ${ }^{1}$, Danping Yang ${ }^{1}$ \& Changfeng Li ${ }^{1,2}$ \\ ${ }^{1}$ Institute of Mathematics, Shandong University, Jinan, China \\ ${ }^{2}$ School of Economics, Shandong University, Jinan, China
}

Correspondence: Yirang Yuan, Institute of Mathematics, Shandong University, Shanda Nanlu No. 27, Jinan, 250100, China. E-mail: yryuan@sdu.edu.cn

\author{
Received: December 20, 2018 Accepted: January 21, 2019 Online Published: March 15, 2019 \\ doi:10.5539/jmr.v11n2p73 \\ URL: https://doi.org/10.5539/jmr.v11n2p73
}

\begin{abstract}
A kind of second-order implicit upwind fractional steps finite difference method is presented in this paper to numerically simulate the coupled system of enhanced (chemical) oil production in porous media. Some techniques, such as the calculus of variations, energy analysis method, commutativity of the products of difference operators, decomposition of high-order difference operators and the theory of a priori estimates are introduced and optimal order error estimates in $l^{2}$ norm are derived.
\end{abstract}

Keywords: enhanced (chemical) oil production, three-dimensional porous coupled system, second-order implicit upwind fractional steps differences, optimal order $l^{2}$ estimates

\section{Introduction}

A mass of residual crude oil stays in the reservoir after water-flooding exploiting because of the constraint of capillary force preventing the motion and the slight influence of injected water and the undesirable fluidity ratio between displacement phase and driven phase weakening the displacement force. Then it is more important to develop the displacement efficiency. A popular method is considered to add some chemical addition agents such as polymer, surfactant and alkali into the injected mixture, which can improve the flooding efficiency. The polymer can optimize the fluidity of displacement phase, modify the ratio with respect to driven phases, balance the leading edges well, weaken the inner porous layer, and increase the efficiency of displacement and the pressure gradient. Surfactant and alkali can decrease interfacial tensions of different phases, then make the bound oil move and gather (Ewing, Yuan \& Li, 1989; Institute of Mathematics, 1995, 2006, 2011; Yuan et al., 1998; Yuan, 2013; Yuan, Cheng, Yang \& Li, 2014, 20142, 20143).

This paper discusses a second-order upwind fractional steps difference method for numerical simulation of enhanced (chemical) oil production in porous media, and gives the theoretical analysis. Based on the previous mathematical and mechanical theory, the software is accomplished, applied in national oilfields such as Daqing Oilfield and Shengli Oilfield and give rise to important benefits and social value.

The mathematical model is a nonlinear coupled system with initial values and boundary values (Ewing, Yuan \& Li, 1989; Institute of Mathematics, 1995, 2006, 2011; Yuan et al., 1998; Yuan, 2013; Yuan et al., 2014, 20142, 20143):

$$
\begin{gathered}
d(c) \frac{\partial p}{\partial t}+\nabla \cdot \mathbf{u}=q(X, t), X=\left(x_{1}, x_{2}, x_{3}\right)^{T} \in \Omega, t \in J=(0, T], \\
\mathbf{u}=-a(c) \nabla p, \quad X \in \Omega, t \in J, \\
\phi(X) \frac{\partial c}{\partial t}+b(c) \frac{\partial p}{\partial t}+\mathbf{u} \cdot \nabla c-\nabla \cdot(D \nabla c)=g(X, t, c), X \in \Omega, t \in J . \\
\phi(X) \frac{\partial}{\partial t}\left(c s_{\alpha}\right)+\nabla \cdot\left(s_{\alpha} \mathbf{u}-\phi c K_{\alpha} \nabla s_{\alpha}\right)=Q_{\alpha}\left(X, t, c, s_{\alpha}\right), X \in \Omega, t \in J, \alpha=1,2, \cdots, n_{c},
\end{gathered}
$$

where $\Omega$ is a bounded domain, $a(c)=a(X, c)=k(X) \mu(c)^{-1}, d(c)=d(X, c)$, and other notations are explained as follows. $\phi(X)$ denotes the porosity of rock, $k(X)$ denotes the permeability, $\mu(c)$ means the viscosity of fluid, and both $D=D(X)$ and $K_{\alpha}=K_{\alpha}(X)\left(\alpha=1,2, \cdots, n_{c}\right)$ denote the diffusion coefficients. $\mathbf{u}$ is Darcy velocity, $p=p(X, t)$ is the pressure, $c=c(X, t)$ means the saturation of water, and $s_{\alpha}=s_{\alpha}(X, t)$ denotes the concentrations of components. The components denote sorts of chemical agents such as the polymer, surfactant, alkali and other ions, and the number is denoted by $n_{c}$. 
Two different boundary value conditions are considered in this paper.

(I) The boundary values condition for the constant pressure:

$$
\begin{gathered}
p=e(X, t), X \in \partial \Omega, t \in J, \\
c=h(X, t), X \in \partial \Omega, t \in J, \\
s_{\alpha}=h_{\alpha}(X, t), X \in \partial \Omega, t \in J, \alpha=1,2, \cdots, n_{c},
\end{gathered}
$$

where $\partial \Omega$ denotes the outer boundary surface of $\Omega$.

(II) The boundary values condition for no permeation case:

$$
\begin{aligned}
& \mathbf{u} \cdot \gamma=0, X \in \partial \Omega, t \in J \\
& D \nabla c \cdot \gamma=0, X \in \partial \Omega, t \in J, \\
& K_{\alpha} \nabla s_{\alpha} \cdot \gamma=0, X \in \partial \Omega, t \in J, \alpha=1,2, \cdots, n_{c},
\end{aligned}
$$

where $\gamma$ denotes the outer normal unit vector.

Initial conditions:

$$
\begin{aligned}
& p(X, 0)=p_{0}(X), X \in \Omega, \\
& c(X, 0)=c_{0}(X), X \in \Omega, \\
& s_{\alpha}(X, 0)=s_{\alpha, 0}(X), X \in \Omega, \alpha=1,2, \cdots, n_{c} .
\end{aligned}
$$

It is easy to compute the concentration by rewriting (3) as the following expression

$$
\phi c \frac{\partial}{\partial t}\left(s_{\alpha}\right)+\mathbf{u} \cdot \nabla s_{\alpha}-\nabla \cdot\left(\phi c K_{\alpha} \nabla s_{\alpha}\right)=Q_{\alpha}-s_{\alpha}\left(q-d(c) \frac{\partial p}{\partial t}+\phi \frac{\partial c}{\partial t}\right), X \in \Omega, t \in J, \alpha=1,2, \cdots, n_{c} .
$$

Under an assumption of periodic condition, Douglas, Ewing, Wheeler, Russell and other scholars present finite difference method and finite element method to analyze a type of two dimensional incompressible two-phase displacement problems and give theoretical error estimates (Douglas, 1981, 1983; Douglas \& Russell, 1982; Ewing, Russell \& Wheeler, 1984). A combination of the characteristic method with normal finite difference method or with normal finite element method is discussed, which can reflect the hyperbolic nature of one-order part of convection-diffusion equations and decrease the order of truncation error. This technique can also overcome numerical oscillation and dispersion, and can improve greatly the computational stability and accuracy. Douglas, Yuan and Ewing present mathematical model of slight compression, numerical method and theoretical analysis for two-dimensional compressible displacement problem under periodic condition and begin a new modern numerical model research (Douglas \& Roberts, 19832; Ewing, 1983; Yuan, 1992, 1993). The authors drop the period condition, give a new modified characteristic finite difference algorithm and finite element algorithm, and derive optimal order error estimates in $L^{2}$-norm (Yuan, 1994, 1996, 19962). An interpolation computation is introduced to deal the points along the characteristics lying outside the bounded domain. The characteristics intersects the grid boundary and the corresponding values of unknown function should be computed, so the time step should vary due to the position of the grids nearby the boundary along characteristics in program design. In conclusion, the actual computation is most complicated (Yuan, 1996, 19962).

For parabolic equations, Axelsson, Ewing, and Lazarov present upwind finite differences (Axelsson \& Gustafasson, 1979; Ewing, Lazarov \& Vassilevski, 1994; Lazarov, Mishev \& Vassilevski, 1996). It can overcome numerical oscillation and cancel extra interpolation computation of grids nearby the boundary along characteristics. Douglas and Peaceman apply upwind method successfully in incompressible two-phase (water and oil) displacements (Peaceman, 1980). While it is hard naturally to give theoretical analysis. The stability and convergence are derived by Fourier method only for constant coefficient cases and this analysis is not generalized for variable coefficient equations (Douglas \& Gunn, 1963, 1964; Marchuk, 1990). Considering actual application, numerical stability and accuracy, the authors present one second-order upwind fractional steps finite difference method for three-dimensional compressible two-phase displacement coupled problem of enhanced oil production. This algorithm can overcome numerical oscillation and dispersion, and decrease the computational scale by decomposition three-dimensional problem into three successive one-dimensional subproblems. Using the calculus of variation, energy analysis method, commutativity of the products of difference operators, decomposition of high-order difference operators and the theory of a priori estimates, the authors give the second-order convergence result of accuracy and error estimates in $l^{2}$-norm, and successfully solve the famous problem of Douglas and Ewing.

Generally, the problem is positive definite,

$$
\begin{aligned}
& 0<a_{*} \leq a(c) \leq a^{*}, 0<d_{*} \leq d(c) \leq d^{*}, 0<\phi_{*} \leq \phi(X) \leq \phi^{*}, \\
& 0<D_{*} \leq D(X, t) \leq D^{*}, 0<K_{*} \leq K_{\alpha}(X) \leq K^{*}, \alpha=1,2, \cdots, n_{c}, \\
& \left|\frac{\partial a}{\partial c}(X, c)\right| \leq A^{*},
\end{aligned}
$$


where $a_{*}, a^{*}, d_{*}, d^{*}, \phi_{*}, \phi^{*}, D_{*}, D^{*} K_{*}, K^{*}$ and $A^{*}$ are positive constants. The coefficients $d(c), b(c), g(c)$ and $Q_{\alpha}\left(c, s_{\alpha}\right)$ are Lipschitz continuous with respect to the unknown functions nearby their $\varepsilon_{0}$ neighbors.

Exact solutions of (1) (6) are assumed to be suitably smooth,

$$
p, c, s_{\alpha} \in L^{\infty}\left(W^{4, \infty}\right) \cap W^{1, \infty}\left(W^{1, \infty}\right), \quad \frac{\partial^{2} p}{\partial t^{2}}, \frac{\partial^{2} c}{\partial t^{2}}, \frac{\partial^{2} s_{\alpha}}{\partial t^{2}} \in L^{\infty}\left(L^{\infty}\right), \alpha=1,2, \cdots, n_{c} .
$$

In this paper, $M$ and $\varepsilon$ denote a general positive constant and a general positive small constant, respectively, and they may have different meanings at different places.

\section{Method}

Let $\Omega_{h}$ denote the partition of $\Omega$ (see Figure 1.). Let $h_{1}, h_{2}$ and $h_{3}$ be three different spacial steps in $x_{1}$-axis, $x_{2}$-axis and $x_{3}$-axis, respectively, and the grid points is denoted by $x_{1 i}=i h_{1}, x_{2 j}=j h_{2}, x_{3 k}=k h_{3}$.

$$
\Omega_{h}=\left\{\begin{array}{l|l}
\left(x_{1 i}, x_{2 j}, x_{3 k}\right) & \begin{array}{l}
i_{1}(j, k)<i<i_{2}(j, k) \\
j_{1}(u, k)<j<j_{2}(i, k) \\
k_{1}(i, j)<k<k_{2}(i, j)
\end{array}
\end{array}\right\}
$$

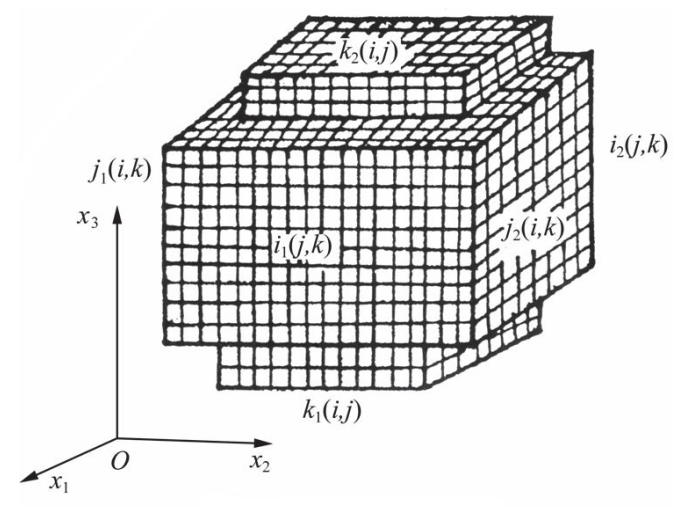

Figure 1. Sketch of the partition of $\Omega_{h}$

Let $\partial \Omega_{h}$ represent the boundary of $\Omega_{h}$ and let $X=\left(x_{1}, x_{2}, x_{3}\right)^{T}, X_{i j k}=\left(i h_{1}, j h_{2}, k h_{3}\right)^{T}, t^{n}=n \Delta t, W\left(X_{i j k}, t^{n}\right)=W_{i j k}^{n}$,

$$
A_{i+1 / 2, j k}^{n}=\left[a\left(X_{i j k}, C_{i j k}^{n}\right)+a\left(X_{i+1, j k}, C_{i+1, j k}^{n}\right)\right] / 2, \quad a_{i+1 / 2, j k}^{n}=\left[a\left(X_{i j k}, c_{i j k}^{n}\right)+a\left(X_{i+1, j k}, c_{i+1, j k}^{n}\right)\right] / 2,
$$

and the signs $A_{i, j+1 / 2, k}^{n}, a_{i, j+1 / 2, k}^{n}, A_{i j, k+1 / 2}^{n}, a_{i j, k+1 / 2}^{n}$ can be defined analogously. Let

$$
\begin{aligned}
& \delta_{\bar{x}_{1}}\left(A^{n} \delta_{x_{1}} P^{n+1}\right)_{i j k}=h_{1}^{-2}\left[A_{i+1 / 2, j k}^{n}\left(P_{i+1, j k}^{n+1}-P_{i j k}^{n+1}\right)-A_{i-1 / 2, j k}^{n}\left(P_{i j k}^{n+1}-P_{i-1, j k}^{n+1}\right)\right], \\
& \delta_{\bar{x}_{2}}\left(A^{n} \delta_{x_{2}} P^{n+1}\right)_{i j k}=h_{2}^{-2}\left[A_{i, j+1 / 2, k}^{n}\left(P_{i, j+1, k}^{n+1}-P_{i j k}^{n+1}\right)-A_{i, j-1 / 2, k}^{n}\left(P_{i j k}^{n+1}-P_{i, j-1, k}^{n+1}\right)\right], \\
& \delta_{\bar{x}_{3}}\left(A^{n} \delta_{x_{3}} P^{n+1}\right)_{i j k}=h_{3}^{-2}\left[A_{i j, k+1 / 2}^{n}\left(P_{i j, k+1}^{n+1}-P_{i j k}^{n+1}\right)-A_{i j, k-1 / 2}^{n}\left(P_{i j k}^{n+1}-P_{i j, k-1}^{n+1}\right)\right], \\
& \nabla_{h}\left(A^{n} \nabla_{h} P^{n+1}\right)_{i j k}=\delta_{\bar{x}_{1}}\left(A^{n} \delta_{x_{1}} P^{n+1}\right)_{i j k}+\delta_{\bar{x}_{2}}\left(A^{n} \delta_{x_{2}} P^{n+1}\right)_{i j k}+\delta_{\bar{x}_{3}}\left(A^{n} \delta_{x_{3}} P^{n+1}\right)_{i j k} .
\end{aligned}
$$


The fractional steps algorithm of flow equation (1) is given by

$$
\begin{aligned}
& d\left(C_{i j k}^{n}\right) \frac{P_{i j k}^{n+1 / 3}-P_{i j k}^{n}}{\Delta t}=\delta_{\bar{x}_{1}}\left(A^{n} \delta_{x_{1}} P^{n+1 / 3}\right)_{i j k}+\delta_{\bar{x}_{2}}\left(A^{n} \delta_{x_{2}} P^{n}\right)_{i j k} \\
& +\delta_{\bar{x}_{3}}\left(A^{n} \delta_{x_{3}} P^{n}\right)_{i j k}+q\left(X_{i j k}, t^{n+1}\right), i_{1}(j, k)<i<i_{2}(j, k), \\
& P_{i j k}^{n+1 / 3}=e_{i j k}^{n+1}, X_{i j k} \in \partial \Omega_{h}, \\
& d\left(C_{i j k}^{n}\right) \frac{P_{i j k}^{n+2 / 3}-P_{i j k}^{n+1 / 3}}{\Delta t}=\delta_{\bar{x}_{2}}\left(A^{n} \delta_{x_{2}}\left(P^{n+2 / 3}-P^{n}\right)\right)_{i j k}, j_{1}(i, k)<j<j_{2}(i, k), \\
& P_{i j k}^{n+2 / 3}=e_{i j k}^{n+1}, X_{i j k} \in \partial \Omega_{h} \text {, } \\
& d\left(C_{i j k}^{n}\right) \frac{P_{i j k}^{n+1}-P_{i j k}^{n+2 / 3}}{\Delta t}=\delta_{\bar{x}_{3}}\left(A^{n} \delta_{x_{3}}\left(P^{n+1}-P^{n}\right)\right)_{i j k}, k_{1}(i, j)<k<k_{2}(i, j), \\
& P_{i j k}^{n+1}=e_{i j k}^{n+1}, X_{i j k} \in \partial \Omega_{h} \text {. }
\end{aligned}
$$

Then the values of Darcy velocity $\mathbf{U}^{\mathbf{n}+\mathbf{1}}=\left(U_{1}^{n+1}, U_{2}^{n+1}, U_{3}^{n+1}\right)^{T}$ are computed by

$$
U_{1, i j k}^{n+1}=-\frac{1}{2}\left[A_{i+1 / 2, j k}^{n} \frac{P_{i+1, j k}^{n+1}-P_{i j k}^{n+1}}{h_{1}}+A_{i-1 / 2, j k}^{n} \frac{P_{i j k}^{n+1}-P_{i-1, j k}^{n+1}}{h_{1}}\right],
$$

and $U_{2, i j k}^{n+1}, U_{3, i j k}^{n+1}$ are obtained similarly.

The implicit upwind fractional steps method of saturation equation (2) is considered.

$$
\begin{aligned}
& \phi_{i j k} \frac{C_{i j k}^{n+1 / 3}-C_{i j k}^{n}}{\Delta t}=\left(1+\frac{h_{1}}{2}\left|U_{1}^{n+1}\right| D^{-1}\right)_{i j k}^{-1} \delta_{\bar{x}_{1}}\left(D \delta_{x_{1}} C^{n+1 / 3}\right)_{i j k} \\
& +\left(1+\frac{h_{2}}{2}\left|U_{2}^{n+1}\right| D^{-1}\right)_{i j k}^{-1} \delta_{\bar{x}_{2}}\left(D \delta_{x_{2}} C^{n}\right)_{i j k}+\left(1+\frac{h_{3}}{2}\left|U_{3}^{n+1}\right| D^{-1}\right)_{i j k}^{-1} \delta_{\bar{x}_{3}}\left(D \delta_{x_{3}} C^{n}\right)_{i j k} \\
& -b\left(C_{i j k}^{n}\right) \frac{P_{i j k}^{n+1}-P_{i j k}^{n}}{\Delta t}+f\left(X_{i j k}, t^{n}, C_{i j k}^{n}\right), i_{1}(j, k)<i<i_{2}(j, k), \\
& C_{i j k}^{n+1 / 3}=h_{i j k}^{n+1}, X_{i j k} \in \partial \Omega_{h}, \\
& \phi_{i j k} \frac{C_{i j k}^{n+2 / 3}-C_{i j k}^{n+1 / 3}}{\Delta t}=\left(1+\frac{h_{2}}{2}\left|U_{2}^{n+1}\right| D^{-1}\right)_{i j k}^{-1} \delta_{\bar{x}_{2}}\left(D \delta_{x_{2}}\left(C^{n+2 / 3}-C^{n}\right)\right)_{i j k}, j_{1}(i, k)<j<j_{2}(i, k), \\
& C_{i j k}^{n+2 / 3}=h_{i j k}^{n+1}, X_{i j k} \in \partial \Omega_{h}, \\
& \phi_{i j k} \frac{C_{i j k}^{n+1}-C_{i j k}^{n+2 / 3}}{\Delta t}=\left(1+\frac{h_{3}}{2}\left|U_{3}^{n+1}\right| D^{-1}\right)_{i j k}^{-1} \delta_{\bar{x}_{3}}\left(D \delta_{x_{3}}\left(C^{n+1}-C^{n}\right)\right)_{i j k} \\
& -\sum_{\beta=1}^{3} \delta_{U_{\beta}^{n+1}, x_{\beta}} C_{i j k}^{n+1}, k_{1}(i, j)<k<k_{2}(i, j), \\
& C_{i j k}^{n+1}=h_{i j k}^{n+1}, X_{i j k} \in \partial \Omega_{h},
\end{aligned}
$$

where $\delta_{U_{1}^{n+1}, x_{1}} C_{i j k}^{n+1}=U_{1, i j k}^{n+1}\left\{H\left(U_{1, i j k}^{n+1}\right) D_{i j k}^{-1} D_{i-1 / 2, j k} \delta_{\bar{x}_{1}}+\left(1-H\left(U_{1, i j k}^{n+1}\right)\right) D_{i j k}^{-1} D_{i+1 / 2, j k} \delta_{x_{1}}\right\} C_{i j k}^{n+1}$,

$\delta_{U_{2}^{n+1, x_{2}}} C_{i j k}^{n+1}=U_{2, i j k}^{n+1}\left\{H\left(U_{2, i j k}^{n+1}\right) D_{i j k}^{-1} D_{i, j-1 / 2, k} \delta_{\bar{x}_{2}}+\left(1-H\left(U_{2, i j k}^{n+1}\right)\right) D_{i j k}^{-1} D_{i, j+1 / 2, k} \delta_{x_{2}}\right\} C_{i j k}^{n+1}$,

$\delta_{U_{3}^{n+1}, x_{3}} C_{i j k}^{n+1}=U_{3, i j k}^{n+1}\left\{H\left(U_{3, i j k}^{n+1}\right) D_{i j k}^{-1} D_{i j, k-1 / 2} \delta_{\bar{x}_{3}}+\left(1-H\left(U_{3, i j k}^{n+1}\right)\right) D_{i j k}^{-1} D_{i j, k+1 / 2} \delta_{x_{3}}\right\} C_{i j k}^{n+1}$, and

$H(z)= \begin{cases}1, & z \geq 0 \\ 0, & z<0\end{cases}$

An implicit upwind fractional steps method, second-order accuracy, of components concentration equation (7) runs in 
parallel, $D_{\alpha}(c)=\phi(X) c(X, t) K_{\alpha}(X)$,

$$
\begin{aligned}
& \phi_{i j k} C_{i j k}^{n+1} \frac{S_{\alpha, i j k}^{n+1 / 3}-S_{\alpha, i j k}^{n}}{\Delta t}=\left(1+\frac{h_{1}}{2}\left|\tilde{U}_{1}^{n+1}\right| D_{\alpha}^{-1}\left(C^{n+1}\right)\right)_{i j k}^{-1} \delta_{\bar{x}_{1}}\left(D_{\alpha}\left(C^{n+1}\right) \delta_{x_{1}} S_{\alpha}^{n+1 / 3}\right)_{i j k} \\
& +\left(1+\frac{h_{2}}{2}\left|\tilde{U}_{2}^{n+1}\right| D_{\alpha}^{-1}\left(C^{n+1}\right)\right)_{i j k}^{-1} \delta_{\bar{x}_{2}}\left(D_{\alpha}\left(C^{n+1}\right) \delta_{x_{2}} S_{\alpha}^{n}\right)_{i j k} \\
& +\left(1+\frac{h_{3}}{2}\left|\tilde{U}_{3}^{n+1}\right| D_{\alpha}^{-1}\left(C^{n+1}\right)\right)_{i j k}^{-1} \delta_{\bar{x}_{3}}\left(D_{\alpha}\left(C^{n+1}\right) \delta_{x_{3}} S_{\alpha}^{n}\right)_{i j k}+Q_{\alpha}\left(C_{i j k}^{n+1}, S_{\alpha, i j k}^{n}\right) \\
& -S_{\alpha, i j k}^{n}\left(q\left(C_{i j k}^{n+1}\right)-d\left(C_{i j k}^{n+1}\right) \frac{P_{i j k}^{n+1}-P_{i j k}^{n}}{\Delta t}+\phi_{i j k} \frac{C_{i j k}^{n+1}-C_{i j k}^{n}}{\Delta t}\right), i_{1}(j, k)<i<i_{2}(j, k), \alpha=1,2, \cdots, n_{c}, \\
& S_{\alpha, i j k}^{n+1 / 3}=h_{\alpha, i j k}^{n+1}, X_{i j k} \in \partial \Omega_{h}, \alpha=1,2, \cdots, n_{c}, \\
& \phi_{i j k} C_{i j k}^{n+1} \frac{S_{\alpha, i j k}^{n+2 / 3}-S_{\alpha, i j k}^{n+1 / 3}}{\Delta t}=\left(1+\frac{h_{2}}{2}\left|\tilde{U}_{2}^{n+1}\right| D_{\alpha}^{-1}\left(C^{n+1}\right)\right)_{i j k}^{-1} \delta_{\bar{x}_{2}}\left(D_{\alpha}\left(C^{n+1}\right) \delta_{x_{2}}\left(S_{\alpha}^{n+2 / 3}-S_{\alpha}^{n}\right)\right)_{i j k}, \\
& S_{\alpha, i j k}^{n+2 / 3}=h_{\alpha, i j k}^{n+1}, X_{i j k} \in \partial \Omega_{h}, \alpha=1,2, \cdots, n_{c}, \\
& \phi_{i j k} C_{i j k}^{n+1} \frac{S_{\alpha, i j k}^{n+1}-S_{\alpha, i j k}^{n+2 / 3}}{\Delta t}=\left(1+\frac{h_{3}}{2}\left|\tilde{U}_{3}^{n+1}\right| D_{\alpha}^{-1}\left(C^{n+1}\right)\right)_{i j k}^{-1} \delta_{\bar{x}_{3}}\left(D_{\alpha}\left(C^{n+1}\right) \delta_{x_{3}}\left(S_{\alpha}^{n+1}-S_{\alpha}^{n}\right)\right)_{i j k} \\
& -\sum_{\beta=1}^{3} \delta_{\bar{U}_{\beta}^{n+1}, x_{\beta}} S_{\alpha, i j k}^{n+1}, \quad k_{1}(i, j)<k<k_{2}(i, j), \alpha=1,2, \cdots, n_{c}, \\
& \quad S_{\alpha, i j k}^{n+1}=h_{\alpha, i j k}^{n+1}, X_{i j k} \in \partial \Omega_{h}, \alpha=1,2, \cdots, n_{c},
\end{aligned}
$$

where $\delta_{\tilde{U}_{1}^{n+1}, x_{1}} S_{\alpha, i j k}^{n+1}=\tilde{U}_{1, i j k}^{n+1}\left\{H\left(\tilde{U}_{1, i j k}^{n+1}\right) D_{\alpha}^{-1}\left(C^{n+1}\right)_{i j k} D_{\alpha}\left(C^{n+1}\right)_{i-1 / 2, j k} \delta_{\bar{x}_{1}}+\left(1-H\left(\tilde{U}_{1, i j k}^{n+1}\right)\right) D_{\alpha}^{-1}\left(C^{n+1}\right)_{i j k} D_{\alpha}\left(C^{n+1}\right)_{i+1 / 2, j k} \delta_{x_{1}}\right\} S_{\alpha, i j k}^{n+1}$, and $\delta_{\tilde{U}_{2}^{n+1}, x_{2}} S_{\alpha, i j k}^{n+1}, \delta_{\tilde{U}_{3}^{n+1}, x_{3}} S_{\alpha, i j k}^{n+1}$ are defined analogously. $\bar{U}_{1, i j k}^{n+1}=\frac{1}{2}\left[A_{i+1 / 2, j k}^{n+1} \frac{P_{i+1, j k}^{n+1}-P_{i j k}^{n+1}}{h_{1}}+A_{i-1 / 2, j k}^{n+1} \frac{P_{i j k}^{n+1}-P_{i-1, j k}^{n+1}}{h_{1}}\right]$, and similarly for $\bar{U}_{2, i j k}^{n+1}, \bar{U}_{3, i j k}^{n+1}$.

Initial approximation:

$$
P_{i j k}^{0}=p_{0}\left(X_{i j k}\right), C_{i j k}^{0}=c_{0}\left(X_{i j k}\right), S_{\alpha, i j k}^{0}=s_{\alpha, 0}\left(X_{i j k}\right), X_{i j k} \in \Omega_{h}, \alpha=1,2, \cdots, n_{c} .
$$

The implicit program runs in the following order. Given $\left\{P_{i j k}^{n}, C_{i j k}^{n}, S_{\alpha, i j k}^{n}, \alpha=1,2, \cdots, n_{c}\right\}$, the solution of transition sheaf $\left\{P_{i j k}^{n+1 / 3}\right\}$ in $x_{1}$ direction is first computed by using the method of speedup from (11a) and (11b), then the transitional solution $\left\{P_{i j k}^{n+2 / 3}\right\}$ in $x_{2}$ direction is computed according to (11c) and (11d), finally the solution $\left\{P_{i j k}^{n+1}\right\}$ in $x_{3}$ direction is obtained according to (11e) and (11f) similarly. Secondly, the values of Darcy velocity $\left\{\mathbf{U}_{i j k}^{n+1}\right\}$ are computed by (12). The computation of saturation proceeds later. The transitional solution $\left\{C_{i j k}^{n+1 / 3}\right\}$ is computed by using the method of speedup in $x_{1}$ direction according to (13a) and (13b), then the transitional solution $\left\{C_{i j k}^{n+2 / 3}\right\}$ in $x_{2}$ direction is computed by (13c) and (13d), finally the solution $\left\{C_{i j k}^{n+1}\right\}$ in $x_{3}$ direction is obtained by (13e) and (13f) similarly. Provided $\left\{C_{i j k}^{n+1}\right\}$, numerical solutions $\left\{\tilde{\mathbf{U}}_{i j k}^{n+1}\right\}$ are obtained continuously. In the last process the values of concentration are computed in parallel for $\alpha=1,2, \cdots, n_{c}$. The transitional solution $\left\{S_{\alpha, i j k}^{n+1 / 3}\right\}$ in $x_{1}$ direction is computed first by using the method of speedup according to (14a) and (14b), then $\left\{S_{\alpha, i j k}^{n+2 / 3}\right\}$ is computed in $x_{2}$ direction by (14c) and (14d), finally the solution $\left\{S_{\alpha, i j k}^{n+1}\right\}$ is obtained by (14e) and (14f) in $x_{3}$ direction analogously. This finite difference solution of (11), (13) and (14) exists, and is unique according to the positive definite condition.

\section{Convergence Analysis}

For convenience, take the domain $\Omega=\{[0,1]\}^{3}, h=1 / N, X_{i j k}=\left(x_{1 i}, x_{2 j}, x_{3 k}\right)^{T}=(i h, j h, k h)^{T}, t^{n}=n \Delta t, W\left(X_{i j k}, t^{n}\right)=W_{i j k}^{n}$ Let $\pi=p-P, \xi=c-C, \zeta_{\alpha}=s_{\alpha}-S_{\alpha}$, where $p, c$ and $s_{\alpha}$ are exact solutions of (1) (6), and $P, C$ and $S_{\alpha}$ are numerical solutions of (11) (14). Introduce inner products and norms in $L^{2}(\Omega)$ and $H^{1}(\Omega)$ (Yuan, 2010, 2012).

$$
<v^{n}, w^{n}>=\sum_{i, j, k=1}^{N} v_{i j k}^{n} w_{i j k}^{n} h^{3},\left\|v^{n}\right\|_{0}^{2}=<v^{n}, v^{n}>,\left[v^{n}, w^{n}\right)_{1}=\sum_{i=0}^{N-1} \sum_{j, k=1}^{N} v_{i j k}^{n} w_{i j k}^{n} h^{3},
$$




$$
\begin{gathered}
{\left[v^{n}, w^{n}\right)_{2}=\sum_{j=0}^{N-1} \sum_{i, k=1}^{N} v_{i j k}^{n} w_{i j k}^{n} h^{3}, \quad\left[v^{n}, w^{n}\right)_{3}=\sum_{k=0}^{N-1} \sum_{i, j=1}^{N} v_{i j k}^{n} w_{i j k}^{n} h^{3},} \\
\|\left[\delta _ { x _ { 1 } } v ^ { n } \| ^ { 2 } = [ \delta _ { x _ { 1 } } v ^ { n } , \delta _ { x _ { 1 } } v ^ { n } ) _ { 1 } , \| \left[\delta _ { x _ { 2 } } v ^ { n } \| ^ { 2 } = [ \delta _ { x _ { 2 } } v ^ { n } , \delta _ { x _ { 2 } } v ^ { n } ) _ { 2 } , \| \left[\delta_{x_{3}} v^{n} \|^{2}=\left[\delta_{x_{3}} v^{n}, \delta_{x_{3}} v^{n}\right)_{3} .\right.\right.\right.
\end{gathered}
$$

Theorem 1 Assume exact solutions of (1) (6) are suitably smooth: $p, c \in W^{1, \infty}\left(W^{1, \infty}\right) \cap L^{\infty}\left(W^{4, \infty}\right), s_{\alpha} \in W^{1, \infty}\left(W^{1, \infty}\right) \cap$ $L^{\infty}\left(W^{4, \infty}\right), \frac{\partial p}{\partial t}, \frac{\partial c}{\partial t} \in L^{\infty}\left(W^{4, \infty}\right), \frac{\partial s_{\alpha}}{\partial t} \in L^{\infty}\left(W^{4, \infty}\right), \frac{\partial^{2} p}{\partial t^{2}} \frac{\partial^{2} c}{\partial t^{2}}, \frac{\partial^{2} s_{\alpha}}{\partial t^{2}} \in L^{\infty}\left(L^{\infty}\right), \alpha=1,2, \cdots, n_{c}$. And assume that the partition parameters satisfy

$$
\Delta t \leq M h^{2}
$$

The difference algorithm (11) (14) are applied layer by layer, then error estimates hold

$$
\begin{aligned}
& \|p-P\|_{\bar{L}^{\infty}\left((0, T] ; h^{1}\right)}+\|c-C\|_{\bar{L}^{\infty}\left((0, T] ; h^{1}\right)}+\left\|d_{t}(p-P)\right\|_{\bar{L}^{2}\left((0, T] ; l^{2}\right)}+\left\|d_{t}(c-C)\right\|_{\bar{L}^{2}\left((0, T] ; l^{2}\right)} \\
& \quad \leq M_{1}^{*}\left\{\Delta t+h^{2}\right\}, \\
& \left\|s_{\alpha}-S_{\alpha}\right\|_{\bar{L}^{\infty}\left((0, T] ; h^{1}\right)}+\left\|d_{t}\left(s_{\alpha}-S_{\alpha}\right)\right\|_{\bar{L}^{2}\left((0, T] ; l^{2}\right)} \leq M_{2}^{*}\left\{\Delta t+h^{2}\right\}, \alpha=1,2, \cdots, n_{c},
\end{aligned}
$$

where $\|g\|_{\bar{L}^{\infty}(J ; \mathcal{M})}=\sup _{n \Delta t \leq T}\left\|g^{n}\right\|_{\mathcal{M}}$, and the constants are $M_{1}^{*}=M_{1}^{*}\|p\|_{W^{1, \infty}\left(W^{4, \infty}\right)},\|p\|_{L^{\infty}\left(W^{4, \infty}\right)}$, $\left.\left\|\frac{\partial p}{\partial t}\right\|_{L^{\infty}\left(W^{4, \infty}\right)},\left\|\frac{\partial^{2} p}{\partial t^{2}}\right\|_{L^{\infty}\left(L^{\infty}\right)},\|c\|_{W^{1, \infty}\left(W^{4, \infty}\right)},\left\|\frac{\partial c}{\partial t}\right\|_{L^{\infty}\left(W^{4, \infty}\right)},\left\|\frac{\partial^{2} c}{\partial t^{2}}\right\|_{L^{\infty}\left(L^{\infty}\right)}\right), M_{2}^{*}=M_{2}^{*}\left(\left\|s_{\alpha}\right\|_{W^{1, \infty}\left(W^{4, \infty}\right)},\left\|\frac{\partial s_{\alpha}}{\partial t}\right\|_{L^{\infty}\left(W^{4, \infty}\right)},\left\|\frac{\partial^{2} s_{\alpha}}{\partial t^{2}}\right\|_{L^{\infty}\left(L^{\infty}\right)}\right)$.

Proof. Considering the flow equation first, cancelling the transitional solutions $P^{n+1 / 3}$ and $P^{n+2 / 3}$ by (11a), (11c) and (11e),

$$
\begin{aligned}
& d\left(C_{i j k}^{n}\right) \frac{P_{i j k}^{n+1}-P_{i j k}^{n}}{\Delta t}-\nabla_{h}\left(A^{n} \nabla_{h} P^{n+1}\right)_{i j k} \\
& =q\left(X_{i j k}, t^{n+1}\right)-(\Delta t)^{2}\left\{\delta_{\bar{x}_{1}}\left(A^{n} \delta_{x_{1}}\left(d^{-1}\left(C^{n}\right) \delta_{x_{2}}\left(A^{n} \delta_{x_{2}} d_{t} P^{n}\right)\right)\right)_{i j k}\right. \\
& \left.+\delta_{\bar{x}_{1}}\left(A^{n} \delta_{x_{1}}\left(d^{-1}\left(C^{n}\right) \delta_{x_{3}}\left(A^{n} \delta_{x_{3}} d_{t} P^{n}\right)\right)\right)_{i j k}+\delta_{\bar{x}_{2}}\left(A^{n} \delta_{x_{2}}\left(d^{-1}\left(C^{n}\right) \delta_{x_{3}}\left(A^{n} \delta_{x_{3}} d_{t} P^{n}\right)\right)\right)_{i j k}\right\} \\
& +(\Delta t)^{3} \delta_{\bar{x}_{1}}\left(A^{n} \delta_{x_{1}}\left(d^{-1}\left(C^{n}\right) \delta_{x_{2}}\left(A^{n} \delta_{x_{2}}\left(d^{-1}\left(C^{n}\right) \delta_{x_{3}}\left(A^{n} \delta_{x_{3}} d_{t} P^{n}\right)\right)\right)\right)\right)_{i j k}, 1 \leq i, j, k \leq N-1, \\
& P_{i j k}^{n+1}=e_{i j k}^{n+1}, X_{i j k} \in \partial \Omega_{h},
\end{aligned}
$$

where $d_{t} P_{i j k}^{n}=\left(P_{i j k}^{n+1}-P_{i j k}^{n}\right) / \Delta t$.

Eq. (18) subtracted from the flow equation $(1)\left(t=t^{n+1}\right)$, it gives rise to the error equation of the pressure,

$$
\begin{aligned}
& d\left(C_{i j k}^{n}\right) \frac{\pi_{i j k}^{n+1}-\pi_{i j k}^{n}}{\Delta t}-\nabla_{h}\left(A^{n} \nabla_{h} \pi^{n+1}\right)_{i j k}=-\left[d\left(c_{i j k}^{n+1}\right)-d\left(C_{i j k}^{n}\right)\right] \frac{p_{i j k}^{n+1}-p_{i j k}^{n}}{\Delta t}+\nabla_{h}\left(\left[a\left(c^{n+1}\right)\right.\right. \\
& \left.\left.-a\left(C^{n}\right)\right] \nabla_{h} p^{n+1}\right)_{i j k}-(\Delta t)^{2}\left\{\left[\delta_{\bar{x}_{1}}\left(a^{n+1} \delta_{x_{1}}\left(d^{-1}\left(c^{n+1}\right) \delta_{x_{2}}\left(a^{n+1} \delta_{x_{2}} d_{t} p^{n}\right)\right)\right)_{i j k}\right.\right. \\
& \left.-\delta_{\bar{x}_{1}}\left(A^{n} \delta_{x_{1}}\left(d^{-1}\left(C^{n}\right) \delta_{x_{2}}\left(A^{n} \delta_{x_{2}} d_{t} P^{n}\right)\right)\right)_{i j k}\right]+\cdots \\
& \left.+\left[\delta_{\bar{x}_{2}}\left(a^{n+1} \delta_{x_{2}}\left(d^{-1}\left(c^{n+1}\right) \delta_{x_{3}}\left(a^{n+1} \delta_{x_{3}} d_{t} p^{n}\right)\right)\right)_{i j k}-\delta_{\bar{x}_{2}}\left(A^{n} \delta_{x_{2}}\left(d^{-1}\left(C^{n}\right) \delta_{x_{3}}\left(A^{n} \delta_{x_{3}} d_{t} P^{n}\right)\right)\right)_{i j k}\right]\right\} \\
& +(\Delta t)^{3}\left\{\delta_{\bar{x}_{1}}\left(a^{n+1} \delta_{x_{1}}\left(d^{-1}\left(c^{n+1}\right) \delta_{x_{2}}\left(a^{n+1} \delta_{x_{2}}\left(d^{-1}\left(c^{n+1}\right) \delta_{x_{3}}\left(a^{n+1} \delta_{x_{3}} d_{t} p^{n}\right)\right)\right)\right)\right)_{i j k}\right. \\
& -\delta_{\bar{x}_{1}}\left(A^{n} \delta_{x_{1}}\left(d^{-1}\left(C^{n}\right) \delta_{x_{2}}\left(A^{n} \delta_{x_{2}}\left(d^{-1}\left(C^{n}\right) \delta_{x_{3}}\left(A^{n} \delta_{x_{3}} d_{t} P^{n}\right)\right)\right)\right)\right)_{i j k}+\sigma_{i j k}^{n+1}, 1 \leq i, j, k \leq N-1, \\
& \pi_{i j k}^{n+1}=0, X_{i j k} \in \partial \Omega_{h},
\end{aligned}
$$

where $\left|\sigma_{i j k}^{n+1}\right| \leq M\left(\left\|\frac{\partial^{2} p}{\partial t^{2}}\right\|_{L^{\infty}\left(L^{\infty}\right)},\left\|\frac{\partial p}{\partial t}\right\|_{L^{\infty}\left(W^{4, \infty}\right)},\|p\|_{L^{\infty}\left(W^{4, \infty}\right)},\|c\|_{L^{\infty}\left(W^{4, \infty}\right)}\right)\left\{\Delta t+h^{2}\right\}$.

An induction hypothesis is given by

$$
\sup _{1 \leq n \leq L} \max \left\{\left\|\pi^{n}\right\|_{1, \infty},\left\|\xi^{n}\right\|_{1, \infty}\right\} \rightarrow 0, \quad(h, \Delta t) \rightarrow 0,
$$

where $\left\|\pi^{n}\right\|_{1, \infty}^{2}=\left|\pi^{n}\right|_{0, \infty}^{2}+\left|\nabla_{h} \pi^{n}\right|_{0, \infty}^{2}$.

Using the calculus of variation, multiplying both sides of error equation (19) by $\delta_{t} \pi_{i j k}^{n}=d_{t} \pi_{i j k}^{n} \Delta t=\pi_{i j k}^{n+1}-\pi_{i j k}^{n}$ and summing by parts, then a form in inner products holds 


$$
\begin{aligned}
& \left\langle d\left(C^{n}\right) d_{t} \pi^{n}, d_{t} \pi^{n}\right\rangle \Delta t+\frac{1}{2}\left\{\left\langle A^{n} \nabla_{h} \pi^{n+1}, \nabla_{h} \pi^{n+1}\right\rangle-\left\langle A^{n} \nabla_{h} \pi^{n}, \nabla_{h} \pi^{n}\right\rangle\right\} \\
& \leq-\left\langle\left[d\left(c^{n+1}\right)-d\left(C^{n}\right)\right] d_{t} p^{n}, d_{t} \pi^{n}\right\rangle \Delta t+\left\langle\nabla_{h}\left(\left[a\left(c^{n+1}\right)-a\left(C^{n}\right)\right] \nabla_{h} p^{n+1}\right), d_{t} \pi^{n}\right\rangle \Delta t \\
& -(\Delta t)^{3}\left\{\left\langle\delta_{\bar{x}_{1}}\left(a^{n+1} \delta_{x_{1}}\left(d^{-1}\left(c^{n+1}\right) \delta_{x_{2}}\left(a^{n+1} \delta_{x_{2}} d_{t} p^{n}\right)\right)\right)-\delta_{\bar{x}_{1}}\left(A^{n} \delta_{x_{1}}\left(d^{-1}\left(C^{n}\right) \delta_{x_{2}}\left(A^{n} \delta_{x_{2}} d_{t} P^{n}\right)\right)\right), d_{t} \pi^{n}\right\rangle\right. \\
& \left.+\cdots+\left\langle\delta_{\bar{x}_{2}}\left(a^{n+1} \delta_{x_{2}}\left(d^{-1}\left(c^{n+1}\right) \delta_{x_{3}}\left(a^{n+1} \delta_{x_{3}} d_{t} p^{n}\right)\right)\right)-\delta_{\bar{x}_{2}}\left(A^{n} \delta_{x_{2}}\left(d^{-1}\left(C^{n}\right) \delta_{x_{3}}\left(A^{n} \delta_{x_{3}} d_{t} P^{n}\right)\right)\right), d_{t} \pi^{n}\right\rangle\right\} \\
& +(\Delta t)^{4}\left\{\left\langle\delta_{\bar{x}_{1}}\left(a^{n+1} \delta_{x_{1}}\left(d^{-1}\left(c^{n+1}\right) \delta_{x_{2}}\left(a^{n+1} \delta_{x_{2}}\left(d^{-1}\left(c^{n+1}\right) \delta_{x_{3}}\left(a^{n+1} \delta_{x_{3}} d_{t} p^{n}\right)\right)\right)\right)\right)\right.\right. \\
& \left.\left.-\delta_{\bar{x}_{1}}\left(A^{n} \delta_{x_{1}}\left(d^{-1}\left(C^{n}\right) \delta_{x_{2}}\left(A^{n} \delta_{x_{2}}\left(d^{-1}\left(C^{n}\right) \delta_{x_{3}}\left(A^{n} \delta_{x_{3}} d_{t} P^{n}\right)\right)\right)\right)\right), d_{t} \pi^{n}\right\rangle\right\}+\left\langle\sigma^{n+1}, d_{t} \pi^{n}\right\rangle \Delta t .
\end{aligned}
$$

Continue to estimate the right terms of (21),

$$
\begin{aligned}
& -\left\langle\left[d\left(c^{n+1}\right)-d\left(C^{n}\right)\right] d_{t} p^{n}, d_{t} \pi^{n}\right\rangle \Delta t \leq M\left\{\left\|\xi^{n}\right\|^{2}+(\Delta t)^{2}\right\} \Delta t+\varepsilon\left\|d_{t} \pi^{n}\right\|^{2} \Delta t, \\
& \left\langle\nabla_{h}\left(\left[a\left(c^{n+1}\right)-a\left(C^{n}\right)\right] \nabla_{h} p^{n+1}\right), d_{t} \pi^{n}\right\rangle \Delta t \leq M\left\{\left\|\nabla_{h} \xi^{n}\right\|^{2}+\left\|\xi^{n}\right\|^{2}+(\Delta t)^{2}\right\} \Delta t+\varepsilon\left\|d_{t} \pi^{n}\right\|^{2} \Delta t .
\end{aligned}
$$

The third term of the right side of (21) is considered. The first part is discussed here

$$
\begin{aligned}
& -(\Delta t)^{3}\left\langle\delta_{\bar{x}_{1}}\left(a^{n+1} \delta_{x_{1}}\left(d^{-1}\left(c^{n+1}\right) \delta_{x_{2}}\left(a^{n+1} \delta_{x_{2}} d_{t} p^{n}\right)\right)\right)-\delta_{\bar{x}_{1}}\left(A^{n} \delta_{x_{1}}\left(d^{-1}\left(C^{n}\right) \delta_{x_{2}}\left(A^{n} \delta_{x_{2}} d_{t} P^{n}\right)\right)\right), d_{t} \pi^{n}\right\rangle \\
& =-(\Delta t)^{3}\left\{\left\langle\delta_{\bar{x}_{1}}\left(A^{n} \delta_{x_{1}}\left(d^{-1}\left(C^{n}\right) \delta_{x_{2}}\left(A^{n} \delta_{x_{2}} d_{t} \pi^{n}\right)\right)\right), d_{t} \pi^{n}\right\rangle+\left\langle\delta _ { \overline { x } _ { 1 } } \left(A ^ { n } \delta _ { x _ { 1 } } \left(d ^ { - 1 } ( C ^ { n } ) \delta _ { x _ { 2 } } \left(\left[ a^{n+1}\right.\right.\right.\right.\right.\right. \\
& \left.\left.\left.\left.\left.-A^{n}\right] \delta_{x_{2}} d_{t} p^{n}\right)\right)\right), d_{t} \pi^{n}\right\rangle+\left\langle\delta_{\bar{x}_{1}}\left(A^{n} \delta_{x_{1}}\left(\left[d^{-1}\left(c^{n+1}\right)-d^{-1}\left(C^{n}\right)\right] \delta_{x_{2}}\left(a^{n+1} \delta_{x_{2}} d_{t} p^{n}\right)\right)\right), d_{t} \pi^{n}\right\rangle \\
& \left.\left.+\left\langle\delta_{\bar{x}_{1}}\left(\left[a^{n+1}-A^{n}\right] \delta_{x_{1}}\left(d^{-1}\left(c^{n+1}\right) \delta_{x_{2}} d_{t} P^{n}\right)\right)\right), d_{t} \pi^{n}\right\rangle\right\} .
\end{aligned}
$$

The operators $-\delta_{\bar{x}_{1}}\left(A^{n} \delta_{x_{1}}\right),-\delta_{\bar{x}_{2}}\left(A^{n} \delta_{x_{2}}\right), \cdots$ are self-conjugate, positive definite, bounded and the domain is a unit cube, but their products are not commutative generally. Noting that the difference operators $\delta_{x_{1}} \delta_{x_{2}}=\delta_{x_{2}} \delta_{x_{1}}, \delta_{\bar{x}_{1}} \delta_{x_{2}}=\delta_{x_{2}} \delta_{\bar{x}_{1}}$, $\delta_{x_{1}} \delta_{\bar{x}_{2}}=\delta_{\bar{x}_{2}} \delta_{x_{1}}, \delta_{\bar{x}_{1}} \delta_{\bar{x}_{2}}=\delta_{\bar{x}_{2}} \delta_{\bar{x}_{1}}$ are commutative, the first term of the right side of (22c) is written by

$$
\begin{aligned}
& -(\Delta t)^{3}\left\langle\delta_{\bar{x}_{1}}\left(A^{n} \delta_{x_{1}}\left(d^{-1}\left(C^{n}\right) \delta_{x_{2}}\left(A^{n} \delta_{x_{2}} d_{t} \pi^{n}\right)\right)\right), d_{t} \pi^{n}\right\rangle \\
& =(\Delta t)^{3}\left\langle d^{-1}\left(C^{n}\right) \delta_{x_{1}} \delta_{x_{2}}\left(A^{n} \delta_{x_{2}} d_{t} \pi^{n}\right)+\delta_{x_{1}} d^{-1}\left(C^{n}\right) \delta_{x_{2}}\left(A^{n} \delta_{x_{2}} d_{t} \pi^{n}\right), A^{n} \delta_{\bar{x}_{1}} d_{t} \pi^{n}\right\rangle \\
& =(\Delta t)^{3}\left\{\left\langle\delta_{\bar{x}_{2}} \delta_{x_{1}}\left(A^{n} \delta_{x_{2}} d_{t} \pi^{n}\right), d^{-1}\left(C^{n}\right) A^{n} \delta_{x_{1}} d_{t} \pi^{n}\right\rangle+\left\langle\delta_{\bar{x}_{2}}\left(A^{n} \delta_{\bar{x}_{2}} d_{t} \pi^{n}\right), \delta_{x_{1}} d^{-1}\left(C^{n}\right) A^{n} \delta_{\bar{x}_{1}} d_{t} \pi^{n}\right\rangle\right\} \\
& \left.=-(\Delta t)^{3}\left\{\left\langle\delta_{\bar{x}_{1}}\left(A^{n} \delta_{x_{2}} d_{t} \pi^{n}\right), \delta_{x_{2}}\left(d^{-1}\left(C^{n}\right) A^{n} \delta_{x_{1}} d_{t} \pi^{n}\right)\right\rangle+\left\langle A^{n} \delta_{x_{2}} d_{t} \pi^{n}\right), \delta_{x_{2}}\left(\delta_{x_{1}} d^{-1}\left(C^{n}\right) A^{n} \delta_{x_{1}} d_{t} \pi^{n}\right)\right\rangle\right\} \\
& =-(\Delta t)^{3}\left\{\left\langle A^{n} \delta_{x_{1}} \delta_{x_{2}} d_{t} \pi^{n}+\delta_{x_{1}} A^{n} \delta_{x_{2}} d_{t} \pi^{n}, d^{-1}\left(C^{n}\right) A^{n} \delta_{x_{1}} \delta_{x_{2}} d_{t} \pi^{n}+\delta_{x_{2}}\left(d^{-1}\left(C^{n}\right) A^{n}\right) \delta_{x_{1}} d_{t} \pi^{n}\right\rangle\right. \\
& \left.+\left\langle A^{n} \delta_{x_{2}} d_{t} \pi^{n}, \delta_{x_{2}} \delta_{x_{1}} d^{-1}\left(C^{n}\right) A^{n} \delta_{x_{1}} d_{t} \pi^{n}+\delta_{x_{1}} d^{-1}\left(C^{n}\right) \delta_{x_{2}} A^{n} \delta_{x_{1}} d_{t} \pi^{n}+\delta_{x_{1}} d^{-1}\left(C^{n}\right) A^{n} \delta_{x_{1}} \delta_{x_{2}} d_{t} \pi^{n}\right\rangle\right\} \\
& =-(\Delta t)^{3} \sum_{i, j, k=1}^{N}\left\{A_{i, j+1 / 2, k}^{n} A_{i+1 / 2, j k}^{n} d^{-1}\left(C_{i j k}^{n}\right)\left[\delta_{x_{1}} \delta_{x_{2}} d_{t} \pi_{i j k}^{n}\right]^{2}\right. \\
& +\left[A_{i, j+1 / 2, k}^{n} \delta_{x_{2}}\left(A_{i+1 / 2, j k}^{n} d^{-1}\left(C_{i j k}^{n}\right)\right) \delta_{x_{1}} d_{t} \pi_{i j k}^{n}+A_{i+1 / 2, j k}^{n} d^{-1}\left(C_{i j k}^{n}\right) \delta_{x_{1}} A_{i, j+1 / 2, k}^{n} \delta_{x_{2}} d_{t} \pi_{i j k}^{n}\right. \\
& \left.+A_{i, j+1 / 2, k}^{n} A_{i+1 / 2, j k}^{n} \delta_{x_{1}} d^{-1}\left(C_{i j k}^{n}\right) \delta_{x_{2}} d_{t} \pi_{i j k}^{n}\right] \delta_{x_{1}} \delta_{x_{2}} d_{t} \pi_{i j k}^{n}+\left[\delta_{x_{1}} A_{i, j+1 / 2, k}^{n} \delta_{x_{2}}\left(d^{-1}\left(C_{i j k}^{n}\right) A_{i+1 / 2, j k}^{n}\right)\right. \\
& \left.\left.+A_{i, j+1 / 2, k}^{n} \delta_{x_{2}} A_{i+1 / 2, j k}^{n} \delta_{x_{1}} d^{-1}\left(C_{i j k}^{n}\right)+A_{i, j+1 / 2, k}^{n} A_{i+1 / 2, j k}^{n} \delta_{x_{1}} \delta_{x_{2}} d^{-1}\left(C_{i j k}^{n}\right)\right] \delta_{x_{2}} d_{t} \pi_{i j k}^{n} \delta_{x_{1}} d_{t} \pi_{i j k}^{n}\right\} h^{3} .
\end{aligned}
$$

By the induction hypothesis (20) we can get that $A_{i+1 / 2, j k}^{n}, A_{i, j+1 / 2, k}^{n}, d^{-1}\left(C_{i j k}^{n}\right), \delta_{x_{2}}\left(A_{i+1 / 2, j k}^{n} d^{-1}\left(C_{i j k}^{n}\right)\right), \delta_{x_{1}} A_{i, j+1 / 2, k}^{n}$ are bounded. Applying the positive definiteness of $A, d^{-1}$ and the decomposition of high order difference operators, we can extract high-order difference quotient term $\delta_{x_{1}} \delta_{x_{2}} d_{t} \pi^{n}$ from the former two terms of the above expression, and cancel related terms by Cauchy inequality,

$$
\begin{aligned}
& -(\Delta t)^{3} \sum_{i, j, k=1}^{N}\left\{A_{i, j+1 / 2, k}^{n} A_{i+1 / 2, j k}^{n} d^{-1}\left(C_{i j k}^{n}\right)\left[\delta_{x_{1}} \delta_{x_{2}} d_{t} \pi_{i j k}^{n}\right]^{2}\right. \\
& +\left[A_{i, j+1 / 2, k}^{n} \delta_{x_{2}}\left(A_{i+1 / 2, j k}^{n} d^{-1}\left(C_{i j k}^{n}\right)\right) \delta_{x_{1}} d_{t} \pi_{i j k}^{n}+A_{i+1 / 2, j k}^{n} d^{-1}\left(C_{i j k}^{n}\right) \delta_{x_{1}} A_{i, j+1 / 2, k}^{n} \delta_{x_{2}} d_{t} \pi_{i j k}^{n}\right. \\
& \left.+\cdots] \delta_{x_{1}} \delta_{x_{2}} d_{t} \pi_{i j k}^{n}\right\} h^{3} \\
& \leq-(\Delta t)^{3} \sum_{i, j, k=1}^{N}\left\{a_{*}^{2}\left(d^{*}\right)^{-1}\left[\delta_{x_{1}} \delta_{x_{2}} d_{t} \pi_{i j k}^{n}\right]^{2}+\left[A_{i, j+1 / 2, k}^{n} \delta_{x_{2}}\left(A_{i+1 / 2, j k}^{n} d^{-1}\left(C_{i j k}^{n}\right)\right) \delta_{x_{1}} d_{t} \pi_{i j k}^{n}\right.\right. \\
& \left.+\cdots] \delta_{x_{1}} \delta_{x_{2}} d_{t} \pi_{i j k}^{n}\right\} h^{3} \leq M\left\{\left\|\delta_{x_{1}} d_{t} \pi^{n}\right\|^{2}+\mid\left[\delta_{x_{2}} d_{t} \pi^{n} \|^{2}\right\}(\Delta t)^{3}\right. \\
& \leq M \Delta t\left\{\left\|\nabla_{h} \pi^{n+1}\right\|^{2}+\left\|\nabla_{h} \pi^{n}\right\|^{2}\right\} .
\end{aligned}
$$


For the third term of (23),

$$
\begin{aligned}
& \text { - }(\Delta t)^{3} \sum_{i, j, k=1}^{N}\left[\delta_{x_{1}} A_{i, j+1 / 2, k}^{n} \delta_{x_{2}}\left(d^{-1}\left(C_{i j k}^{n}\right) A_{i+1 / 2, j k}^{n}\right)\right. \\
& \left.+A_{i, j+1 / 2, k}^{n} \delta_{x_{2}} A_{i+1 / 2, j k}^{n} \delta_{x_{1}} d^{-1}\left(C_{i j k}^{n}\right)\right] \delta_{x_{1}} d_{t} \pi_{i j k}^{n} \delta_{x_{2}} d_{t} \pi_{i j k}^{n} h^{3} \\
& \leq M\left\{\left\|\nabla_{h} \pi^{n+1}\right\|^{2}+\left\|\nabla_{h} \pi^{n}\right\|^{2}\right\} \Delta t, \\
& -(\Delta t)^{3} \sum_{i, j, k=1}^{N} A_{i, j+1 / 2, k}^{n} A_{i+1 / 2, j k}^{n} \delta_{x_{1}} \delta_{x_{2}} d^{-1}\left(C_{i j k}^{n}\right) \delta_{x_{1}} d_{t} \pi_{i j k}^{n} \delta_{x_{2}} d_{t} \pi_{i j k}^{n} h^{3} \\
& \leq M(\Delta t)^{1 / 2}\left\|d_{t} \pi^{n}\right\|^{2} \Delta t \leq \varepsilon\left\|d_{t} \pi^{n}\right\|^{2} \Delta t,
\end{aligned}
$$

Then,

$$
\begin{aligned}
& -(\Delta t)^{3}\left\{\left\langle\delta_{\bar{x}_{1}}\left(A^{n} \delta_{x_{1}}\left(d^{-1}\left(C^{n}\right) \delta_{x_{2}}\left(A^{n} \delta_{x_{2}} d_{t} \pi^{n}\right)\right)\right), d_{t} \pi^{n}\right\rangle\right. \\
& \leq M\left\{\left\|\nabla_{h} \pi^{n+1}\right\|^{2}+\left\|\nabla_{h} \pi^{n}\right\|^{2}\right\} \Delta t+\varepsilon\left\|d_{t} \pi^{n}\right\|^{2} \Delta t .
\end{aligned}
$$

Considering the rest of (22c) similarly,

$$
\begin{aligned}
& -(\Delta t)^{3}\left\langle\delta_{\bar{x}_{1}}\left(a^{n+1} \delta_{x_{1}}\left(d^{-1}\left(c^{n+1}\right) \delta_{x_{2}}\left(a^{n+1} \delta_{x_{2}} d_{t} p^{n}\right)\right)\right)-\delta_{\bar{x}_{1}}\left(A^{n} \delta_{x_{1}}\left(d^{-1}\left(C^{n}\right) \delta_{x_{2}}\left(A^{n} \delta_{x_{2}} d_{t} P^{n}\right)\right)\right), d_{t} \pi^{n}\right\rangle \\
& \leq M\left\{\left\|\nabla_{h} \pi^{n+1}\right\|^{2}+\left\|\nabla_{h} \pi^{n}\right\|^{2}+\left\|\xi^{n}\right\|^{2}+(\Delta t)^{2}\right\} \Delta t+\varepsilon\left\|d_{t} \pi^{n}\right\|^{2} \Delta t .
\end{aligned}
$$

The estimates (26) can be obtained analogously for the other two terms of the third right term of (21).

For the fourth term on the right hand side of (21),

$$
\begin{aligned}
& (\Delta t)^{4}\left\{\left\langle\delta_{\bar{x}_{1}}\left(A^{n} \delta_{x_{1}}\left(d^{-1}\left(C^{n}\right) \delta_{x_{2}}\left(A^{n} \delta_{x_{2}}\left(d^{-1}\left(C^{n}\right) \delta_{x_{3}}\left(A^{n} \delta_{x_{3}} d_{t} \pi^{n}\right)\right)\right)\right)\right), d_{t} \pi^{n}\right\rangle+\cdots\right\} \\
& \leq-\frac{1}{2} a_{*}^{3}\left(d^{*}\right)^{-2}(\Delta t)^{4} \sum_{i, j, k=1}^{N}\left[\delta_{x_{1}} \delta_{x_{2}} \delta_{x_{3}} d_{t} \pi_{i j k}^{n}\right]^{2} h^{3} \\
& \quad+M\left\{\left\|\nabla_{h} \pi^{n+1}\right\|^{2}+\left\|\nabla_{h} \pi^{n}\right\|^{2}+\left\|\xi^{n}\right\|^{2}+(\Delta t)^{2}\right\} \Delta t+\varepsilon\left\|d_{t} \pi^{n}\right\|^{2} \Delta t .
\end{aligned}
$$

Collecting (22) (27), it holds for error equation (21) as $\Delta t$ and $\varepsilon$ are sufficiently small,

$$
\begin{aligned}
& \left\|d_{t} \pi^{n}\right\|^{2} \Delta t+\frac{1}{2}\left\{\left\langle A^{n} \nabla_{h} \pi^{n+1}, \nabla_{h} \pi^{n+1}\right\rangle-\left\langle A^{n} \nabla_{h} \pi^{n}, \nabla_{h} \pi^{n}\right\rangle\right\} \\
& \leq M\left\{\left\|\nabla_{h} \pi^{n+1}\right\|^{2}+\left\|\nabla_{h} \pi^{n}\right\|^{2}+h^{4}+(\Delta t)^{2}\right\} \Delta t .
\end{aligned}
$$


Error estimates of the saturation equation is discussed later. Cancelling $C_{i j k}^{n+1 / 3}$ and $C_{i j k}^{n+2 / 3}$ of (13a), (13c) and (13e),

$$
\begin{aligned}
& \phi_{i j k} \frac{C_{i j k}^{n+1}-C_{i j k}^{n}}{\Delta t}-\sum_{\beta=1}^{3}\left(1+\frac{h}{2}\left|U_{\beta}^{n+1}\right| D^{-1}\right)_{i j k}^{-1} \delta_{\bar{x}_{\beta}}\left(D \delta_{x_{\beta}} C^{n+1}\right)_{i j k} \\
& =-\sum_{\beta=1}^{3} \delta_{U_{\beta}^{n+1}, x_{\beta}} C_{i j k}^{n+1}-b\left(C_{i j k}^{n}\right) \frac{P_{i j k}^{n+1}-P_{i j k}^{n}}{\Delta t}+g\left(X_{i j k}, t^{n}, C_{i j k}^{n}\right) \\
& -(\Delta t)^{2}\left\{\left(1+\frac{h}{2}\left|U_{1}^{n+1}\right| D^{-1}\right)_{i j k}^{-1} \delta_{\bar{x}_{1}}\left(D \delta_{x_{1}}\left[\phi^{-1}\left(1+\frac{h}{2}\left|U_{2}^{n+1}\right| D^{-1}\right)^{-1} \delta_{\bar{x}_{2}}\left(D \delta_{x_{2}} d_{t} C^{n}\right)\right]\right)_{i j k}\right. \\
& +\left(1+\frac{h}{2}\left|U_{1}^{n+1}\right| D^{-1}\right)_{i j k}^{-1} \delta_{\bar{x}_{1}}\left(D \delta_{x_{1}}\left[\phi^{-1}\left(1+\frac{h}{2}\left|U_{3}^{n+1}\right| D^{-1}\right)^{-1} \delta_{\bar{x}_{3}}\left(D \delta_{x_{3}} d_{t} C^{n}\right)\right]\right)_{i j k} \\
& \left.+\left(1+\frac{h}{2}\left|U_{2}^{n+1}\right| D^{-1}\right)_{i j k}^{-1} \delta_{\bar{x}_{2}}\left(D \delta_{x_{2}}\left[\phi^{-1}\left(1+\frac{h}{2}\left|U_{3}^{n+1}\right| D^{-1}\right)^{-1} \delta_{\bar{x}_{3}}\left(D \delta_{x_{3}} d_{t} C^{n}\right)\right]\right)_{i j k}\right\} \\
& +(\Delta t)^{3}\left(1+\frac{h}{2}\left|U_{1}^{n+1}\right| D^{-1}\right)_{i j k}^{-1} \delta_{\bar{x}_{1}}\left(D \delta _ { x _ { 1 } } \left[\phi ^ { - 1 } ( 1 + \frac { h } { 2 } | U _ { 2 } ^ { n + 1 } | D ^ { - 1 } ) ^ { - 1 } \delta _ { \overline { x } _ { 2 } } \left(D \delta _ { x _ { 2 } } \left[\phi^{-1}\left(1+\frac{h}{2}\left|U_{3}^{n+1}\right| D^{-1}\right)^{-1}\right.\right.\right.\right. \\
& \left.\left.\delta_{\bar{x}_{3}}\left(D \delta_{x_{3}} d_{t} C^{n}\right)\right]\right)_{i j k}+\Delta t\left\{\left(1+\frac{h}{2}\left|U_{1}^{n+1}\right| D^{-1}\right)_{i j k}^{-1} \delta_{\bar{x}_{1}}\left(D \delta_{x_{1}}\left(\phi^{-1} \sum_{\beta=1}^{3} \delta_{U_{\beta}^{n+1}, x_{\beta}} C^{n+1}\right)\right)_{i j k}\right. \\
& \left.+\left(1+\frac{h}{2}\left|U_{2}^{n+1}\right| D^{-1}\right)_{i j k}^{-1} \delta_{\bar{x}_{2}}\left(D \delta_{x_{2}}\left(\phi^{-1} \sum_{\beta=1}^{3} \delta_{U_{\beta}^{n+1}, x_{\beta}} C^{n+1}\right)\right)_{i j k}\right\}-(\Delta t)^{2}\left(1+\frac{h}{2}\left|U_{1}^{n+1}\right| D^{-1}\right)_{i j k}^{-1} \\
& \delta_{\bar{x}_{1}}\left(D \delta_{x_{1}}\left(\phi^{-1}\left(1+\frac{h}{2}\left|U_{2}^{n+1}\right| D^{-1}\right)_{i j k}^{-1} \delta_{\bar{x}_{2}}\left(D \delta_{x_{2}}\left(\phi^{-1} \sum_{\beta=1}^{3} \delta_{U_{\beta}^{n+1}, x_{\beta}} C^{n+1}\right)\right)\right)\right)_{i j k}, 1 \leq i, j, k \leq N-1, \\
& C_{i j k}^{n+1}=h_{i j k}^{n+1}, X_{i j k} \in \partial \Omega_{h},
\end{aligned}
$$

Error equation of the saturation is obtained by (2) $\left(t=t^{n+1}\right)$ and (29)

$$
\begin{aligned}
& \phi_{i j k} \frac{\xi_{i j k}^{n+1}-\xi_{i j k}^{n}}{\Delta t}-\sum_{\beta=1}^{3}\left(1+\frac{h}{2}\left|u_{\beta}^{n+1}\right| D^{-1}\right)_{i j k}^{-1} \delta_{\bar{x}_{\beta}}\left(D \delta_{x_{\beta}} \xi^{n+1}\right)_{i j k}=\sum_{\beta=1}^{3}\left\{\delta_{U_{\beta}^{n+1}, x_{\beta}} C_{i j k}^{n+1}-\delta_{u_{\beta}^{n+1}, x_{\beta}} c_{i j k}^{n+1}\right\} \\
& +\sum_{\beta=1}^{3}\left[\left(1+\frac{h}{2}\left|u_{\beta}^{n+1}\right| D^{-1}\right)_{i j k}^{-1}-\left(1+\frac{h}{2}\left|U_{\beta}^{n+1}\right| D^{-1}\right)_{i j k}^{-1}\right] \delta_{\bar{x}_{\beta}}\left(D \delta_{x_{\beta}} C^{n+1}\right)_{i j k} \\
& +g\left(X_{i j k}, t^{n+1}, c_{i j k}^{n+1}\right)-g\left(X_{i j k}, t^{n}, C_{i j k}^{n}\right)-b\left(C_{i j k}^{n} \frac{\pi_{i j k}^{n+1}-\pi_{i j k}^{n}}{\Delta t}-\left[b\left(c_{i j k}^{n+1}\right)-b\left(C_{i j k}^{n}\right)\right] \frac{p_{i j k}^{n+1}-p_{i j k}^{n}}{\Delta t}\right. \\
& -(\Delta t)^{2}\left\{\left[\left(1+\frac{h}{2}\left|u_{1}^{n+1}\right| D^{-1}\right)_{i j k}^{-1} \delta_{\bar{x}_{1}}\left(D \delta_{x_{1}}\left[\phi^{-1}\left(1+\frac{h}{2}\left|u_{2}^{n+1}\right| D^{-1}\right)^{-1} \delta_{\bar{x}_{2}}\left(D \delta_{x_{2}} d_{t} c^{n}\right)\right]\right)_{i j k}\right.\right. \\
& \left.-\left(1+\frac{h}{2}\left|U_{1}^{n+1}\right| D^{-1}\right)_{i j k}^{-1} \delta_{\bar{x}_{1}}\left(D \delta_{x_{1}}\left[\phi^{-1}\left(1+\frac{h}{2}\left|U_{2}^{n+1}\right| D^{-1}\right)^{-1} \delta_{\bar{x}_{2}}\left(D \delta_{x_{2}} d_{t} C^{n}\right)\right]\right)_{i j k}\right] \\
& +\cdots+\left[\left(1+\frac{h}{2}\left|u_{2}^{n+1}\right| D^{-1}\right)_{i j k}^{-1} \delta_{\bar{x}_{2}}\left(D \delta_{x_{2}}\left[\phi^{-1}\left(1+\frac{h}{2}\left|u_{3}^{n+1}\right| D^{-1}\right)^{-1} \delta_{\bar{x}_{3}}\left(D \delta_{x_{3}} d_{t} c^{n}\right)\right]\right)_{i j k}\right.
\end{aligned}
$$




$$
\begin{aligned}
& \left.\left.-\left(1+\frac{h}{2}\left|U_{2}^{n+1}\right| D^{-1}\right)_{i j k}^{-1} \delta_{\bar{x}_{2}}\left(D \delta_{x_{2}}\left[\phi^{-1}\left(1+\frac{h}{2}\left|U_{3}^{n+1}\right| D^{-1}\right)^{-1} \delta_{\bar{x}_{3}}\left(D \delta_{x_{3}} d_{t} C^{n}\right)\right]\right)_{i j k}\right]\right\} \\
& +(\Delta t)^{3}\left\{( 1 + \frac { h } { 2 } | u _ { 1 } ^ { n + 1 } | D ^ { - 1 } ) _ { i j k } ^ { - 1 } \delta _ { \overline { x } _ { 1 } } \left(D \delta _ { x _ { 1 } } \left[\phi ^ { - 1 } ( 1 + \frac { h } { 2 } | u _ { 2 } ^ { n + 1 } | D ^ { - 1 } ) ^ { - 1 } \delta _ { \overline { x } _ { 2 } } \left(D \delta _ { x _ { 2 } } \left[\phi^{-1}\left(1+\frac{h}{2}\left|u_{3}^{n+1}\right| D^{-1}\right)^{-1}\right.\right.\right.\right.\right. \\
& \left.\left.\delta_{\bar{x}_{3}}\left(D \delta_{x_{3}} d_{t} c^{n}\right)\right]\right)_{i j k}-\left(1+\frac{h}{2}\left|U_{1}^{n+1}\right| D^{-1}\right)_{i j k}^{-1} \delta_{\bar{x}_{1}}\left(D \delta _ { x _ { 1 } } \left[\phi ^ { - 1 } ( 1 + \frac { h } { 2 } | U _ { 2 } ^ { n + 1 } | D ^ { - 1 } ) ^ { - 1 } \delta _ { \overline { x } _ { 2 } } \left(D \delta_{x_{2}} \mid \phi^{-1}(1+\right.\right.\right. \\
& \left.\left.\left.\left.+\frac{h}{2}\left|U_{3}^{n+1}\right| D^{-1}\right)^{-1} \delta_{\bar{x}_{3}}\left(D \delta_{x_{3}} d_{t} C^{n}\right)\right]\right)_{i j k}\right\}+\Delta t\left\{\left(1+\frac{h}{2}\left|u_{1}^{n+1}\right| D^{-1}\right)_{i j k}^{-1} \delta_{\bar{x}_{1}}\left(D \delta_{x_{1}}\left(\phi^{-1} \sum_{\beta=1}^{3} \delta_{u_{\beta}^{n+1}, x_{\beta}} c^{n+1}\right)\right)_{i j k}\right. \\
& \left.-\left(1+\frac{h}{2}\left|U_{1}^{n+1}\right| D^{-1}\right)_{i j k}^{-1} \delta_{\bar{x}_{1}}\left(D \delta_{x_{1}}\left(\phi^{-1} \sum_{\beta=1}^{3} \delta_{U_{\beta}^{n+1}, x_{\beta}} C^{n+1}\right)\right)_{i j k}+\cdots\right\} \\
& -(\Delta t)^{2}\left\{\left(1+\frac{h}{2}\left|u_{1}^{n}\right| D^{-1}\right)_{i j k}^{-1} \delta_{\bar{x}_{1}}\left(D \delta_{x_{1}}\left(\phi^{-1}\left(1+\frac{h}{2}\left|u_{2}^{n}\right| D^{-1}\right)_{i j k}^{-1} \delta_{\bar{x}_{2}}\left(D \delta_{x_{2}}\left(\phi^{-1} \sum_{\beta=1}^{3} \delta_{u_{\beta}^{n+1}, x_{\beta}} c^{n+1}\right)\right)\right)\right)_{i j k}\right. \\
& \left.-\left(1+\frac{h}{2}\left|U_{1}^{n+1}\right| D^{-1}\right)_{i j k}^{-1} \delta_{\bar{x}_{1}}\left(D \delta_{x_{1}}\left(\phi^{-1}\left(1+\frac{h}{2}\left|U_{2}^{n+1}\right| D^{-1}\right)_{i j k}^{-1} \delta_{\bar{x}_{2}}\left(D \delta_{x_{2}}\left(\phi^{-1} \sum_{\beta=1}^{3} \delta_{U_{\beta}^{n+1}, x_{\beta}} C^{n+1}\right)\right)\right)\right)_{i j k}\right\} \\
& +\varepsilon_{i j k}^{n+1}, 1 \leq i, j, k \leq N-1, \\
& \xi_{i j k}^{n+1}=0, X_{i j k} \in \partial \Omega_{h},
\end{aligned}
$$

For

$$
\begin{aligned}
& \frac{\partial}{\partial x_{\beta}}\left(D \frac{\partial c^{n+1}}{\partial x_{\beta}}\right)_{i j k}-\left(1+\frac{h}{2}\left|u_{\beta, i j k}^{n+1}\right| D_{i j k}^{-1}\right)^{-1} \delta_{\bar{x}_{\beta}}\left(D \delta_{x_{\beta}} c^{n+1}\right)_{i j k} \\
& =\frac{h}{2}\left|u_{\beta, i j k}^{n+1}\right| D_{i j k}^{-1} \delta_{\bar{x}_{\beta}}\left(D \delta_{x_{\beta}} c^{n+1}\right)_{i j k}+O\left(h^{2}\right), \beta=1,2,3 .
\end{aligned}
$$

Then

$$
\left|\varepsilon_{i j k}^{n+1}\right| \leq M\left(\left\|\frac{\partial^{2} c}{\partial t^{2}}\right\|_{L^{\infty}\left(L^{\infty}\right)},\left\|\frac{\partial c}{\partial t}\right\|_{L^{\infty}\left(W^{4, \infty}\right)},\|c\|_{L^{\infty}\left(W^{4, \infty}\right)},\left\|\frac{\partial p}{\partial t}\right\|_{L^{\infty}\left(W^{4, \infty}\right)}\right)\left\{h^{2}+\Delta t\right\} .
$$

Multiplying both sides of (30) by $\delta_{t} \xi_{i j k}^{n}=\xi_{i j k}^{n+1}-\xi_{i j k}^{n}=d_{t} \xi_{i j k}^{n} \Delta t$ and summing by parts, we can get the inner product expression of error equation

$$
\begin{aligned}
& \left\langle\phi d_{t} \xi^{n}, d_{t} \xi^{n}\right\rangle \Delta t+\sum_{\beta=1}^{3}\left\langle D \delta_{x_{\beta}} \xi^{n+1}, \delta_{x_{\beta}}\left[\left(1+\frac{h}{2}\left|u_{\beta}^{n+1}\right| D^{-1}\right)^{-1}\left(\xi^{n+1}-\xi^{n}\right)\right]\right\rangle \\
& =\sum_{\beta=1}^{3}\left\langle\delta_{U_{\beta}^{n+1}, x_{\beta}} C^{n+1}-\delta_{u_{\beta}^{n+1}, x_{\beta}} c^{n+1}, d_{t} \xi^{n}\right\rangle \Delta t \\
& +\sum_{\beta=1}^{3}\left\langle\left[\left(1+\frac{h}{2}\left|u_{\beta}^{n+1}\right| D^{-1}\right)^{-1}-\left(1+\frac{h}{2}\left|U_{\beta}^{n+1}\right| D^{-1}\right)^{-1}\right] \delta_{\bar{x}_{\beta}}\left(D \delta_{x_{\beta}} C^{n+1}\right), d_{t} \xi^{n}\right\rangle \Delta t \\
& +\left\langle g\left(c^{n+1}\right)-g\left(C^{n}\right), d_{t} \xi^{n}\right\rangle \Delta t-\left\langle b\left(C^{n}\right) \frac{\pi^{n+1}-\pi^{n}}{\Delta t}, d_{t} \xi^{n}\right\rangle \Delta t-\left\langle\left[b\left(c^{n+1}\right)-b\left(C^{n}\right)\right] \frac{p^{n+1}-p^{n}}{\Delta t}, d_{t} \xi^{n}\right\rangle \Delta t \\
& -(\Delta t)^{3}\left\{\left\langle\left(1+\frac{h}{2}\left|U_{1}^{n+1}\right| D^{-1}\right)^{-1} \delta_{\bar{x}_{1}}\left(D \delta_{x_{1}}\left[\phi^{-1}\left(1+\frac{h}{2}\left|U_{2}^{n+1}\right| D^{-1}\right)^{-1} \delta_{\bar{x}_{2}}\left(D \delta_{x_{2}} d_{t} \xi^{n}\right)\right]\right), d_{t} \xi^{n}\right\rangle+\cdots\right. \\
& \left.+\left\langle\left(1+\frac{h}{2}\left|U_{2}^{n+1}\right| D^{-1}\right)^{-1} \delta_{\bar{x}_{2}}\left(D \delta_{x_{2}}\left[\phi^{-1}\left(1+\frac{h}{2}\left|U_{3}^{n+1}\right| D^{-1}\right)^{-1} \delta_{\bar{x}_{3}}\left(D \delta_{x_{3}} d_{t} \xi^{n}\right)\right]\right), d_{t} \xi^{n}\right\rangle+\cdots\right\}+ \\
& +(\Delta t)^{4}\left\{\left\langle( 1 + \frac { h } { 2 } | U _ { 1 } ^ { n + 1 } | D ^ { - 1 } ) ^ { - 1 } \delta _ { \overline { x } _ { 1 } } \left(D \delta _ { x _ { 1 } } \left[\phi ^ { - 1 } ( 1 + \frac { h } { 2 } | U _ { 2 } ^ { n + 1 } | D ^ { - 1 } ) ^ { - 1 } \delta _ { \overline { x } _ { 2 } } \left(D \delta _ { x _ { 2 } } \left[\phi^{-1}\left(1+\frac{h}{2}\left|U_{3}^{n+1}\right| D^{-1}\right)^{-1}\right.\right.\right.\right.\right.\right. \\
& \left.\left.\left.\left.\delta_{\bar{x}_{3}}\left(D \delta_{x_{3}} d_{t} \xi^{n}\right)\right]\right), d_{t} \xi^{n}\right\rangle+\cdots\right\}+(\Delta t)^{2}\left\{\left\langle\left(1+\frac{h}{2}\left|u_{1}^{n+1}\right| D^{-1}\right)^{-1} \delta_{\bar{x}_{1}}\left(D \delta_{x_{1}}\left(\phi^{-1} \sum_{\beta=1}^{3} \delta_{u_{\beta}^{n+1}, x_{\beta}} c^{n+1}\right)\right)\right.\right. \\
& \left.\left.-\left(1+\frac{h}{2}\left|U_{1}^{n+1}\right| D^{-1}\right)^{-1} \delta_{\bar{x}_{1}}\left(D \delta_{x_{1}}\left(\phi^{-1} \sum_{\beta=1}^{3} \delta_{U_{\beta}^{n+1}, x_{\beta}} C^{n+1}\right)\right), d_{t} \xi^{n}\right\rangle+\cdots\right\}
\end{aligned}
$$




$$
\begin{aligned}
& -(\Delta t)^{3}\left\langle\left(1+\frac{h}{2}\left|u_{1}^{n+1}\right| D^{-1}\right)^{-1} \delta_{\bar{x}_{1}}\left(D \delta_{x_{1}}\left(\phi^{-1}\left(1+\frac{h}{2}\left|u_{2}^{n+1}\right| D^{-1}\right)^{-1} \delta_{\bar{x}_{2}}\left(D \delta_{x_{2}}\left(\phi^{-1} \sum_{\beta=1}^{3} \delta_{u_{\beta}^{n+1}, x_{\beta}} c^{n+1}\right)\right)\right)\right),\right. \\
& \left.-\left(1+\frac{h}{2}\left|U_{1}^{n+1}\right| D^{-1}\right)^{-1} \delta_{\bar{x}_{1}}\left(D \delta_{x_{1}}\left(\phi^{-1}\left(1+\frac{h}{2}\left|U_{2}^{n+1}\right| D^{-1}\right)^{-1} \delta_{\bar{x}_{2}}\left(D \delta_{x_{2}}\left(\phi^{-1} \sum_{\beta=1}^{3} \delta_{U_{\beta}^{n+1}, x_{\beta}} C^{n+1}\right)\right)\right)\right), d_{t} \xi^{n}\right\rangle \\
& +\left\langle\varepsilon^{n+1}, d_{t} \xi^{n}\right\rangle \Delta t .
\end{aligned}
$$

For the second term of the left side,

$$
\begin{aligned}
& \left\langle D \delta_{x_{\beta}} \xi^{n+1}, \delta_{x_{\beta}}\left[\left(1+\frac{h}{2}\left|u_{\beta}^{n+1}\right| D^{-1}\right)^{-1}\left(\xi^{n+1}-\xi^{n}\right)\right]\right\rangle \\
& \geq \frac{1}{2}\left\{\left\langle D \delta_{x_{\beta}} \xi^{n+1},\left(1+\frac{h}{2}\left|u_{\beta}^{n+1}\right| D^{-1}\right)^{-1} \delta_{x_{\beta}} \xi^{n+1}\right\rangle-\left\langle D \delta_{x_{\beta}} \xi^{n},\left(1+\frac{h}{2}\left|u_{\beta}^{n+1}\right| D^{-1}\right)^{-1} \delta_{x_{\beta}} \xi^{n}\right\rangle\right\} \\
& -M \mid\left[\delta_{x_{\beta}} \xi^{n+1}\left\|^{2} \Delta t-\varepsilon\right\| d_{t} \xi^{n} \|^{2} \Delta t . \beta=1,2,3 .\right.
\end{aligned}
$$

The other terms on the right side of (31) are considered. The velocity vector $\mathbf{U}^{n+1}$ is bounded by the induction hypothesis (20),

$$
\begin{aligned}
& \sum_{\beta=1}^{3}\left\langle\delta_{U_{\beta}^{n+1}, x_{\beta}} C^{n+1}-\delta_{u_{\beta}^{n+1}, x_{\beta}} c^{n+1}, d_{t} \xi^{n}\right\rangle \Delta t \\
& \leq M\left\{\left\|\mathbf{u}^{n+1}-\mathbf{U}^{n+1}\right\|^{2}+\left\|\nabla_{h} \xi^{n+1}\right\|^{2}+(\Delta t)^{2}\right\} \Delta t+\varepsilon\left\|d_{t} \xi^{n}\right\|^{2} \Delta t .
\end{aligned}
$$

It is to estimate the second term of the right side of (31). Note that

$$
\left(1+\frac{h}{2}\left|u_{\beta, i j k}^{n+1}\right| D_{i j k}^{-1}\right)^{-1}-\left(1+\frac{h}{2}\left|U_{\beta, i j k}^{n+1}\right| D_{i j k}^{-1}\right)^{-1}=\frac{\frac{h}{2}\left(\left|U_{\beta, i j k}^{n+1}\right|-\left|u_{\beta, i j k}^{n+1}\right|\right) D_{i j k}^{-1}}{\left(1+\frac{h}{2}\left|u_{\beta, i j k}^{n+1}\right| D_{i j k}^{-1}\right)\left(1+\frac{h}{2}\left|U_{\beta, i j k}^{n+1}\right| D_{i j k}^{-1}\right)}, \beta=1,2,3,
$$

and (20),

$$
\begin{aligned}
& \sum_{\beta=1}^{3}\left\langle\left[\left(1+\frac{h}{2}\left|u_{\beta}^{n+1}\right| D^{-1}\right)^{-1}-\left(1+\frac{h}{2}\left|U_{\beta}^{n+1}\right| D^{-1}\right)^{-1}\right] \delta_{\bar{x}_{\beta}}\left(D \delta_{x_{\beta}} C^{n+1}\right), d_{t} \xi^{n}\right\rangle \Delta t \\
& \leq M\left\{\left\|\mathbf{u}^{n+1}-\mathbf{U}^{n+1}\right\|^{2}+(\Delta t)^{2}\right\} \Delta t+\varepsilon\left\|d_{t} \xi^{n}\right\|^{2} \Delta t .
\end{aligned}
$$

By $\varepsilon_{0}$-Lipschitz continuity and (20), it is derived for the third, fourth, fifth terms and the last term of the right side of (31),

$$
\begin{aligned}
& \left\langle g\left(c^{n+1}\right)-g\left(C^{n}\right), d_{t} \xi^{n}\right\rangle \Delta t \leq M\left\{\left\|\xi^{n}\right\|^{2}+(\Delta t)^{2}\right\} \Delta t+\varepsilon\left\|d_{t} \xi^{n}\right\|^{2} \Delta t, \\
& -\left\langle b\left(C^{n}\right) \frac{\pi^{n+1}-\pi^{n}}{\Delta t}, d_{t} \xi^{n}\right\rangle \Delta t \leq M\left\|d_{t} \pi^{n}\right\|^{2} \Delta t+\varepsilon\left\|d_{t} \xi^{n}\right\|^{2} \Delta t, \\
& -\left\langle\left[b\left(c^{n+1}\right)-b\left(C^{n}\right)\right] \frac{p^{n+1}-p^{n}}{\Delta t}, d_{t} \xi^{n}\right\rangle \Delta t \leq M\left\{\left\|\xi^{n}\right\|^{2}+(\Delta t)^{2}\right\} \Delta t+\varepsilon\left\|d_{t} \xi^{n}\right\|^{2} \Delta t, \\
& \left\langle\varepsilon^{n+1}, d_{t} \xi^{n}\right\rangle \Delta t \leq M\left\{h^{4}+(\Delta t)^{2}\right\} \Delta t+\varepsilon\left\|d_{t} \xi^{n}\right\|^{2} \Delta t .
\end{aligned}
$$

The sixth term of the right side of (31) is analyzed as follows.

$$
\begin{aligned}
& -(\Delta t)^{3}\left\langle\left(1+\frac{h}{2}\left|U_{1}^{n+1}\right| D^{-1}\right)^{-1} \delta_{\bar{x}_{1}}\left(D \delta_{x_{1}}\left[\phi^{-1}\left(1+\frac{h}{2}\left|U_{2}^{n+1}\right| D^{-1}\right)^{-1} \delta_{\bar{x}_{2}}\left(D \delta_{x_{2}} d_{t} \xi^{n}\right)\right]\right), d_{t} \xi^{n}\right\rangle \\
& =(\Delta t)^{3}\left\langleD \left(\delta_{x_{1}}\left(\phi^{-1}\left(1+\frac{h}{2}\left|U_{2}^{n+1}\right| D^{-1}\right)^{-1}\right) \delta_{\bar{x}_{2}}\left(D \delta_{x_{2}} d_{t} \xi^{n}\right)+\phi^{-1}\left(1+\frac{h}{2}\left|U_{2}^{n+1}\right| D^{-1}\right)^{-1}\right.\right. \\
& \left.\left.\delta_{x_{1}} \delta_{\bar{x}_{2}}\left(D \delta_{x_{2}} d_{t} \xi^{n}\right)\right), \delta_{x_{1}}\left(1+\frac{h}{2}\left|U_{1}^{n+1}\right| D^{-1}\right)^{-1} d_{t} \xi^{n}+\left(1+\frac{h}{2}\left|U_{1}^{n+1}\right| D^{-1}\right)^{-1} \delta_{x_{1}} d_{t} \xi^{n}\right\rangle \\
& =-(\Delta t)^{3}\left\langle\left\langle D \delta_{x_{1}} \delta_{x_{2}} d_{t} \xi^{n}+\delta_{x_{1}} D \delta_{x_{2}} d_{t} \xi^{n}, D \phi^{-1}\left(1+\frac{h}{2}\left|U_{2}^{n+1}\right| D^{-1}\right)^{-1}\left(1+\frac{h}{2}\left|U_{1}^{n+1}\right| D^{-1}\right)^{-1}\right.\right. \\
& \delta_{x_{1}} \delta_{x_{2}} d_{t} \xi^{n}+\left\{\delta_{x_{2}}\left[D \phi^{-1}\left(1+\frac{h}{2}\left|U_{2}^{n+1}\right| D^{-1}\right)^{-1}\left(1+\frac{h}{2}\left|U_{1}^{n+1}\right| D^{-1}\right)^{-1}\right] \delta_{x_{1}} d_{t} \xi^{n}+D \phi^{-1}(1+\right. \\
& \left.+\frac{h}{2}\left|U_{2}^{n+1}\right| D^{-1}\right)^{-1} \delta_{x_{1}}\left(1+\frac{h}{2}\left|U_{1}^{n+1}\right| D^{-1}\right)^{-1} \delta_{x_{2}} d_{t} \xi^{n}+\delta_{x_{2}}\left[D \phi^{-1}\left(1+\frac{h}{2}\left|U_{2}^{n+1}\right| D^{-1}\right)^{-1}\right. \\
& \left.\left.\left.\delta_{x_{1}}\left(1+\frac{h}{2}\left|U_{1}^{n+1}\right| D^{-1}\right)^{-1}\right] d_{t} \xi^{n}\right\}\right\rangle+\left\langle D \delta_{x_{2}} d_{t} \xi^{n}, D \delta_{x_{1}}\left(\phi^{-1}\left(1+\frac{h}{2}\left|U_{2}^{n+1}\right| D^{-1}\right)^{-1}(1+\right.\right.
\end{aligned}
$$




$$
\begin{aligned}
& \left.\left.+\frac{h}{2}\left|U_{1}^{n+1}\right| D^{-1}\right)^{-1}\right) \delta_{x_{1}} \delta_{x_{2}} d_{t} \xi^{n}+\left\{\delta_{x_{2}}\left[D \delta_{x_{1}}\left(\phi^{-1}\left(1+\frac{h}{2}\left|U_{2}^{n+1}\right| D^{-1}\right)^{-1}\right)\left(1+\frac{h}{2}\left|U_{1}^{n+1}\right| D^{-1}\right)^{-1}\right]\right. \\
& \delta_{x_{1}} d_{t} \xi^{n}+D \delta_{x_{1}}\left(\phi^{-1}\left(1+\frac{h}{2}\left|U_{2}^{n+1}\right| D^{-1}\right)^{-1}\right) \delta_{x_{1}}\left(1+\frac{h}{2}\left|U_{1}^{n+1}\right| D^{-1}\right)^{-1} \delta_{x_{2}} d_{t} \xi^{n} \\
& \left.\left.\left.+\delta_{x_{2}}\left[D \delta_{x_{1}}\left(\phi^{-1}\left(1+\frac{h}{2}\left|U_{2}^{n+1}\right| D^{-1}\right)^{-1}\right) \delta_{x_{1}}\left(1+\frac{h}{2}\left|U_{2}^{n+1}\right| D^{-1}\right)^{-1}\right] d_{t} \xi^{n}\right\}\right\rangle\right\} .
\end{aligned}
$$

For the terms of the above expression,

$$
\begin{aligned}
& -(\Delta t)^{3}\left\langle D \delta_{x_{1}} \delta_{x_{2}} d_{t} \xi^{n}, D \phi^{-1}\left(1+\frac{h}{2}\left|U_{2}^{n+1}\right| D^{-1}\right)^{-1}\left(1+\frac{h}{2}\left|U_{1}^{n+1}\right| D^{-1}\right)^{-1} \delta_{x_{1}} \delta_{x_{2}} d_{t} \xi^{n}\right\rangle \\
& =-(\Delta t)^{3} \sum_{i, j, k=1}^{N} D_{i, j-1 / 2, k} D_{i-1 / 2, j k} \phi_{i j k}^{-1}\left(1+\frac{h}{2}\left|U_{2}^{n+1}\right| D^{-1}\right)_{i j k}^{-1}\left(1+\frac{h}{2}\left|U_{1}^{n+1}\right| D^{-1}\right)_{i j k}^{-1}\left(\delta_{x_{1}} \delta_{x_{2}} d_{t} \xi_{i j k}^{n}\right)^{2} h^{3},
\end{aligned}
$$

Note that $0<D_{*} \leq D(X) \leq D^{*}, 0<\phi_{*} \leq \phi(X) \leq \phi^{*}$, and $\mathbf{U}^{n+1}$ is bounded, we have $0<b_{1} \leq\left(1+\frac{h}{2}\left|U_{2}^{n+1}\right| D^{-1}\right)_{i j k}^{-1}$, $0<b_{2} \leq\left(1+\frac{h}{2}\left|U_{1}^{n+1}\right| D^{-1}\right)_{i j k}^{-1}$, then have

$$
\begin{aligned}
& -(\Delta t)^{3}\left\langle D \delta_{x_{1}} \delta_{x_{2}} d_{t} \xi^{n}, D \phi^{-1}\left(1+\frac{h}{2}\left|U_{2}^{n+1}\right| D^{-1}\right)^{-1}\left(1+\frac{h}{2}\left|U_{1}^{n+1}\right| D^{-1}\right)^{-1} \delta_{x_{1}} \delta_{x_{2}} d_{t} \xi^{n}\right\rangle \\
& \leq-(\Delta t)^{3} D_{*}^{2}\left(\phi^{*}\right)^{-1} b_{1} b_{2} \sum_{i, j, k=1}^{N}\left(\delta_{x_{1}} \delta_{x_{2}} d_{t} \xi_{i j k}^{n}\right)^{2} h^{3} .
\end{aligned}
$$

The terms consisting of $\delta_{x_{1}} \delta_{x_{2}} d_{t} \xi^{n}$ are

$$
\begin{aligned}
& -(\Delta t)^{3} \mid\left\langle D \delta_{x_{1}} \delta_{x_{2}} d_{t} \xi^{n}, \delta_{x_{2}}\left[D \phi^{-1}\left(1+\frac{h}{2}\left|U_{2}^{n+1}\right| D^{-1}\right)^{-1}\left(1+\frac{h}{2}\left|U_{1}^{n+1}\right| D^{-1}\right)^{-1}\right] \delta_{x_{1}} d_{t} \xi^{n}\right. \\
& \left.+D \phi^{-1}\left(1+\frac{h}{2}\left|U_{2}^{n+1}\right| D^{-1}\right)^{-1} \delta_{x_{1}}\left(1+\frac{h}{2}\left|U_{1}^{n+1}\right| D^{-1}\right)^{-1} \delta_{x_{2}} d_{t} \xi^{n}\right\rangle \\
& +\left\langle\delta_{x_{1}} D \delta_{x_{2}} d_{t} \xi^{n}, D \phi^{-1}\left(1+\frac{h}{2}\left|U_{2}^{n+1}\right| D^{-1}\right)^{-1}\left(1+\frac{h}{2}\left|U_{1}^{n+1}\right| D^{-1}\right)^{-1} \delta_{x_{1}} \delta_{x_{2}} d_{t} \xi^{n}\right\rangle \\
& \left.+\left\langle D \delta_{x_{2}} d_{t} \xi^{n}, D \delta_{x_{1}}\left(\phi^{-1}\left(1+\frac{h}{2}\left|U_{2}^{n+1}\right| D^{-1}\right)^{-1}\right)\left(1+\frac{h}{2}\left|U_{1}^{n+1}\right| D^{-1}\right)^{-1} \delta_{x_{1}} \delta_{x_{2}} d_{t} \xi^{n}\right\rangle\right\} .
\end{aligned}
$$

The first term is discussed first. It is derived that $h\left\|\mathbf{U}^{n+1}\right\|_{1, \infty}$ is bounded by the induction hypothesis and inverse theorem, and the operators $\delta_{x_{2}}\left(1+\frac{h}{2}\left|U_{2}^{n+1}\right| D^{-1}\right)_{i j k}^{-1}, \delta_{x_{2}}\left(1+\frac{h}{2}\left|U_{1}^{n+1}\right| D^{-1}\right)_{i j k}^{-1}$ are bounded. Applying $\varepsilon$-Cauchy inequality,

$$
\begin{aligned}
& -(\Delta t)^{3}\left\langle D \delta_{x_{1}} \delta_{x_{2}} d_{t} \xi^{n}, \delta_{x_{2}}\left[D \phi^{-1}\left(1+\frac{h}{2}\left|U_{2}^{n+1}\right| D^{-1}\right)^{-1}\left(1+\frac{h}{2}\left|U_{1}^{n+1}\right| D^{-1}\right)^{-1}\right] \delta_{x_{1}} d_{t} \xi^{n}\right\rangle \\
& =-(\Delta t)^{3} \sum_{i, j, k=1}^{N} D_{i, j-1 / 2, k} \delta_{x_{2}}\left[D_{i-1 / 2, j k} \phi_{i j k}^{-1}\left(1+\frac{h}{2}\left|U_{2}^{n+1}\right| D^{-1}\right)_{i j k}^{-1}\left(1+\frac{h}{2}\left|U_{1}^{n+1}\right| D^{-1}\right)_{i j k}^{-1}\right] \\
& \delta_{x_{1}} \delta_{x_{2}} d_{t} \xi_{i j k}^{n} \delta_{x_{1}} d_{t} \xi_{i j k}^{n} h^{3} \\
& \leq \varepsilon(\Delta t)^{3} \sum_{i, j, k=1}^{N}\left(\delta_{x_{1}} \delta_{x_{2}} d_{t} \xi_{i j k}^{n}\right)^{2} h^{3}+M(\Delta t)^{3} \sum_{i, j, k=1}^{N}\left(\delta_{x_{1}} d_{t} \xi^{n}\right)^{2} h^{3} .
\end{aligned}
$$

Estimate the other terms of (37b) similarly.

$$
\begin{aligned}
& -(\Delta t)^{3}\left\{\left\langle\left(1+\frac{h}{2}\left|U_{1}^{n+1}\right| D^{-1}\right)^{-1} \delta_{\bar{x}_{1}}\left(D \delta_{x_{1}}\left[\phi^{-1}\left(1+\frac{h}{2}\left|U_{2}^{n+1}\right| D^{-1}\right)^{-1} \delta_{x_{2}}\left(D \delta_{x_{2}} d_{t} \xi^{n}\right)\right]\right), d_{t} \xi^{n}\right\rangle+\cdots\right\} \\
& \leq M\left\{\left\|\nabla_{h} \xi^{n+1}\right\|^{2}+\left\|\nabla_{h} \xi^{n}\right\|^{2}+\left\|\xi^{n+1}\right\|^{2}+\left\|\xi^{n}\right\|^{2}\right\} \Delta t .
\end{aligned}
$$

The same result (38) can be obtained for the other parts of the sixth term.

For the seventh, eighth and ninth terms, it holds by the condition (16), the induction hypothesis (20) and inverse estimates,

$$
\begin{aligned}
& (\Delta t)^{4}\left\{\left\langle( 1 + \frac { h } { 2 } | U _ { 1 } ^ { n + 1 } | D ^ { - 1 } ) ^ { - 1 } \delta _ { \overline { x } _ { 1 } } \left(D \delta _ { x _ { 1 } } \left[\phi ^ { - 1 } ( 1 + \frac { h } { 2 } | U _ { 2 } ^ { n + 1 } | D ^ { - 1 } ) ^ { - 1 } \delta _ { \overline { x } _ { 2 } } \left(D \delta _ { x _ { 2 } } \left[\phi^{-1}(1+\right.\right.\right.\right.\right.\right. \\
& \left.\left.\left.\left.\left.+\frac{h}{2}\left|U_{3}^{n+1}\right| D^{-1}\right)^{-1} \delta_{\bar{x}_{3}}\left(D \delta_{x_{3}} d_{t} \xi^{n}\right)\right]\right), d_{t} \xi^{n}\right\rangle+\cdots\right\} \\
& \leq \varepsilon\left\|d_{t} \xi^{n}\right\|^{2} \Delta t+M\left\{\left\|\nabla_{h} \xi^{n+1}\right\|^{2}+\left\|\nabla_{h} \xi^{n}\right\|^{2}+\left\|\xi^{n}\right\|^{2}+(\Delta t)^{2}\right\} \Delta t .
\end{aligned}
$$




$$
\begin{aligned}
& (\Delta t)^{2}\left\{\left\langle\left(1+\frac{h}{2}\left|u_{1}^{n+1}\right| D^{-1}\right)^{-1} \delta_{\bar{x}_{1}}\left(D \delta_{x_{1}}\left(\phi^{-1} \sum_{\beta=1}^{3} \delta_{u_{\beta}^{n+1}, x_{\beta}} c^{n+1}\right)\right)\right.\right. \\
& \left.\left.-\left(1+\frac{h}{2}\left|U_{1}^{n+1}\right| D^{-1}\right)^{-1} \delta_{\bar{x}_{1}}\left(D \delta_{x_{1}}\left(\phi^{-1} \sum_{\beta=1}^{3} \delta_{U_{\beta}^{n+1}, x_{\beta}} C^{n+1}\right)\right), d_{t} \xi^{n}\right\rangle+\cdots\right\} \\
& -(\Delta t)^{3}\left\langle\left(1+\frac{h}{2}\left|u_{1}^{n+1}\right| D^{-1}\right)^{-1} \delta_{\bar{x}_{1}}\left(D \delta_{x_{1}}\left(\phi^{-1}\left(1+\frac{h}{2}\left|u_{2}^{n+1}\right| D^{-1}\right)^{-1} \delta_{\bar{x}_{2}}\left(D \delta_{x_{2}}\left(\phi^{-1} \sum_{\beta=1}^{3} \delta_{u_{\beta}^{n+1}, x_{\beta}} c^{n+1}\right)\right)\right)\right)\right. \\
& \left.-\left(1+\frac{h}{2}\left|U_{1}^{n+1}\right| D^{-1}\right)^{-1} \delta_{\bar{x}_{1}}\left(D \delta_{x_{1}}\left(\phi^{-1}\left(1+\frac{h}{2}\left|U_{2}^{n+1}\right| D^{-1}\right)^{-1} \delta_{\bar{x}_{2}}\left(D \delta_{x_{2}}\left(\phi^{-1} \sum_{\beta=1}^{3} \delta_{U_{\beta}^{n+1}, x_{\beta}} C^{n+1}\right)\right)\right)\right), d_{t} \xi^{n}\right\rangle \\
& \leq \varepsilon\left\|d_{t} \xi^{n}\right\|^{2} \Delta t+M\left\{\left\|\mathbf{u}^{n+1}-\mathbf{U}^{n+1}\right\|^{2}+\left\|\nabla_{h} \xi^{n+1}\right\|^{2}+\left\|\nabla_{h} \xi^{n}\right\|^{2}+\left\|\xi^{n+1}\right\|^{2}+\left\|\xi^{n}\right\|^{2}+(\Delta t)^{2}\right\} \Delta t .
\end{aligned}
$$

Applying (38) (40) to estimate (31),

$$
\begin{aligned}
& \left\|d_{t} \xi^{n}\right\|^{2} \Delta t+\frac{1}{2} \sum_{\beta=1}^{3}\left[\left\langle D \delta_{x_{\beta}} \xi^{n+1},\left(1+\frac{h}{2}\left|u_{\beta}^{n+1}\right| D^{-1}\right)^{-1} \delta_{x_{\beta}} \xi^{n+1}\right\rangle-\left\langle D \delta_{x_{\beta}} \xi^{n},\left[\left(1+\frac{h}{2}\left|u_{\beta}^{n+1}\right| D^{-1}\right)^{-1} \delta_{x_{\beta}} \xi^{n}\right\rangle\right]\right. \\
& \leq \varepsilon\left\|d_{t} \xi^{n}\right\|^{2} \Delta t+M\left\{\left\|\mathbf{u}^{n+1}-\mathbf{U}^{n+1}\right\|^{2}+\left\|d_{t} \pi^{n}\right\|^{2}+\left\|\nabla_{h} \xi^{n+1}\right\|^{2}+\left\|\nabla_{h} \xi^{n}\right\|^{2}+\left\|\xi^{n+1}\right\|^{2}\right. \\
& \left.\quad+\left\|\xi^{n}\right\|^{2}+(\Delta t)^{2}\right\} \Delta t
\end{aligned}
$$

Summing the resulting error (28) on $t$ for $0 \leq n \leq L$ and noting that $\pi^{0}=0$,

$$
\begin{aligned}
& \sum_{n=0}^{L}\left\|d_{t} \pi^{n}\right\|^{2} \Delta t+\left\langle A^{L} \nabla_{h} \pi^{L+1}, \nabla_{h} \pi^{L+1}\right\rangle \\
& \leq \sum_{n=1}^{L}\left\langle\left[A^{n}-A^{n-1}\right] \nabla_{h} \pi^{n}, \nabla_{h} \pi^{n}\right\rangle+M \sum_{n=1}^{L}\left\{\left\|\nabla \pi_{h}^{n+1}\right\|^{2}+h^{4}+(\Delta t)^{2}\right\} \Delta t \\
& \leq \varepsilon \sum_{n=1}^{L}\left\|d_{t} \xi^{n-1}\right\|^{2} \Delta t+M \sum_{n=1}^{L}\left\{\left\|\nabla_{h} \pi^{n+1}\right\|^{2}+h^{4}+(\Delta t)^{2}\right\} \Delta t .
\end{aligned}
$$

Summing (41) on $t$ for $0 \leq n \leq L$, and noting that $\xi^{0}=0$ and $\left\|\mathbf{u}^{n+1}-\mathbf{U}^{n+1}\right\|^{2} \leq M\left\{\left\|\xi^{n}\right\|^{2}+\left\|\nabla_{h} \pi^{n+1}\right\|^{2}+h^{4}\right\}$,

$$
\begin{aligned}
& \sum_{n=0}^{L}\left\|d_{t} \xi^{n}\right\|^{2} \Delta t+\frac{1}{2} \sum_{\beta=1}^{3}\left[\left\langle D \delta_{x_{\beta}} \xi^{L+1},\left(1+\frac{h}{2}\left|u_{\beta}^{L+1}\right| D^{-1}\right)^{-1} \delta_{x_{\beta}} \xi^{L+1}\right\rangle-\left\langle D \delta_{x_{\beta}} \xi^{0},\left[\left(1+\frac{h}{2}\left|u_{\beta}^{0}\right| D^{-1}\right)^{-1} \delta_{x_{\beta}} \xi^{0}\right\rangle\right]\right. \\
& \leq \sum_{n=1}^{L}\left\{\sum_{\beta=1}^{3}\left\langle D \delta_{x_{\beta}} \xi^{n},\left[\left(1+\frac{h}{2}\left|u_{\beta}^{n+1}\right| D^{-1}\right)^{-1}-\left(1+\frac{h}{2}\left|u_{\beta}^{n}\right| D^{-1}\right)^{-1}\right] \delta_{x_{\beta}} \xi^{n}\right\rangle\right\} \\
& \quad+\varepsilon\left\|d_{t} \xi^{n}\right\|^{2} \Delta t+M\left\{\left\|d_{t} \pi^{n}\right\|^{2}+\left\|\nabla_{h} \xi^{n+1}\right\|^{2}+\left\|\nabla_{h} \xi^{n}\right\|^{2}+\left\|\xi^{n+1}\right\|^{2}+\left\|\xi^{n}\right\|^{2}+(\Delta t)^{2}\right\} \Delta t
\end{aligned}
$$

Note

$$
\begin{aligned}
& \left|\left(1+\frac{h}{2}\left|u_{\beta, i j k}^{n+1}\right| D_{i j k}^{-1}\right)^{-1}-\left(1+\frac{h}{2}\left|u_{\beta, i j k}^{n}\right| D_{i j k}^{-1}\right)^{-1}\right|=\frac{\left|\frac{h}{2}\left(\left|u_{\beta, i j k}^{n}\right|-\left|u_{\beta, i j k}^{n+1}\right|\right) D_{i j k}^{-1}\right|}{\left(1+\frac{h}{2}\left|u_{\beta, i j k}^{n+1}\right| D_{i j k}^{-1}\right)\left(1+\frac{h}{2}\left|u_{\beta, i j k}^{n}\right| D_{i j k}^{-1}\right)} \\
& \leq \frac{\frac{h}{2} D_{i j k}^{-1}\left|d_{t} u_{\beta, i j k}^{n}\right| \Delta t}{\left(1+\frac{h}{2}\left|u_{\beta, i j k}^{n+1}\right| D_{i j k}^{-1}\right)\left(1+\frac{h}{2}\left|u_{\beta, i j k}^{n}\right| D_{i j k}^{-1}\right)} \leq M h \Delta t, \beta=1,2,3,
\end{aligned}
$$

Combining (43) and (44),

$$
\begin{aligned}
& \sum_{n=0}^{L}\left\|d_{t} \xi^{n}\right\|^{2} \Delta t+\frac{1}{2} \sum_{\beta=1}^{3}\left\langle D \delta_{x_{\beta}} \xi^{L+1},\left(1+\frac{h}{2}\left|u_{\beta}^{L+1}\right| D^{-1}\right)^{-1} \delta_{x_{\beta}} \xi^{L+1}\right\rangle \\
& \leq M \sum_{n=0}^{L}\left\|d_{t} \pi^{n}\right\|^{2} \Delta t+M \sum_{n=0}^{L}\left\{\left\|\nabla_{h} \pi^{n+1}\right\|^{2}+\left\|\nabla_{h} \xi^{n+1}\right\|_{1}^{2}+h^{4}+(\Delta t)^{2}\right\} \Delta t
\end{aligned}
$$


Since the condition $\pi^{0}=\xi^{0}=0$,

$$
\left\|\pi^{L+1}\right\|^{2} \leq \varepsilon \sum_{n=0}^{L}\left\|d_{t} \pi^{n}\right\|^{2} \Delta t+M \sum_{n=0}^{L}\left\|\pi^{n}\right\|^{2} \Delta t, \quad\left\|\xi^{L+1}\right\|^{2} \leq \varepsilon \sum_{n=0}^{L}\left\|d_{t} \xi^{n}\right\|^{2} \Delta t+M \sum_{n=0}^{L}\left\|\xi^{n}\right\|^{2} \Delta t .
$$

Collecting (42) and (45),

$$
\sum_{n=0}^{L}\left[\left\|d_{t} \pi^{n}\right\|^{2}+\left\|d_{t} \xi^{n}\right\|^{2}\right] \Delta t+\left\|\pi^{L+1}\right\|_{1}^{2}+\left\|\xi^{L+1}\right\|_{1}^{2} \leq M \sum_{n=0}^{L}\left\{\left\|\pi^{n+1}\right\|_{1}^{2}+\left\|\xi^{n+1}\right\|_{1}^{2}+h^{4}+(\Delta t)^{2}\right\} \Delta t .
$$

Using the Gronwall lemma,

$$
\sum_{n=0}^{L}\left[\left\|d_{t} \pi^{n}\right\|^{2}+\left\|d_{t} \xi^{n}\right\|^{2}\right] \Delta t+\left\|\pi^{L+1}\right\|_{1}^{2}+\left\|\xi^{L+1}\right\|_{1}^{2} \leq M\left\{h^{4}+(\Delta t)^{2}\right\} .
$$

It remains to test the induction hypothesis (20). It is right as $n=0$ because of $\pi^{0}=\xi^{0}=0$. Assume the induction hypothesis holds for any positive integer $n$ between 1 and a given positive integer $l$. By (47) we have $\left\|\pi^{l+1}\right\|_{1}+\left\|\xi^{l+1}\right\|_{1} \leq$ $M\left\{h^{2}+\Delta t\right\}$. Then by (16) and inverse estimates $\left\|\pi^{l+1}\right\|_{1, \infty}+\left\|\xi^{l+1}\right\|_{1, \infty} \leq M h^{1 / 2},(20)$ holds for $n=l+1$. Therefore, error estimate (17a) has been proved.

Then the error estimates of components concentration are considered. Cancelling the transition solutions $S_{\alpha}^{n+1 / 3}$ and $S_{\alpha}^{n+2 / 3}$ of (14a), (14c) and (14e),

$$
\begin{aligned}
& \phi_{i j k} C_{i j k}^{n+1} \frac{S_{\alpha, i j k}^{n+1}-S_{\alpha, i j k}^{n}}{\Delta t}-\sum_{\beta=1}^{3}\left(1+\frac{h}{2}\left|\tilde{U}_{\beta}^{n+1}\right| D_{\alpha}^{-1}\left(C^{n+1}\right)\right)_{i j k}^{-1} \delta_{\bar{x}_{\beta}}\left(D_{\alpha}\left(C^{n+1}\right) \delta_{x_{\beta}} S_{\alpha}^{n+1}\right)_{i j k} \\
& =-\sum_{\beta=1}^{3} \delta_{\bar{U}_{\beta}^{n+1}, x_{\beta}} S_{\alpha, i j k}^{n+1}+Q_{\alpha}\left(S_{\alpha, i j k}^{n}\right)-S_{\alpha, i j k}^{n}\left(q\left(C_{i j k}^{n+1}\right)-d\left(C_{i j k}^{n+1}\right) \frac{P_{i j k}^{n+1}-P_{i j k}^{n}}{\Delta t}+\phi_{i j k} \frac{C_{i j k}^{n+1}-C_{i j k}^{n}}{\Delta t}\right) \\
& -(\Delta t)^{2}\left\{( 1 + \frac { h } { 2 } | \tilde { U } _ { 1 } ^ { n + 1 } | D _ { \alpha } ^ { - 1 } ( C ^ { n + 1 } ) ) _ { i j k } ^ { - 1 } \delta _ { \overline { x } _ { 1 } } \left(D _ { \alpha } ( C ^ { n + 1 } ) \delta _ { x _ { 1 } } \left(\left(C^{n+1} \phi\right)^{-1}\left(1+\frac{h}{2}\left|\tilde{U}_{2}^{n+1}\right| D_{\alpha}^{-1}\left(C^{n+1}\right)\right)^{-1}\right.\right.\right. \\
& \left.\left.\delta_{\bar{x}_{2}}\left(D_{\alpha}\left(C^{n+1}\right) \delta_{x_{2}} d_{t} S_{\alpha}^{n}\right)\right)\right)_{i j k}+\cdots+\left(1+\frac{h}{2}\left|\tilde{U}_{2}^{n+1}\right| D_{\alpha}^{-1}\left(C^{n+1}\right)\right)_{i j k}^{-1} \delta_{\bar{x}_{2}}\left(D _ { \alpha } ( C ^ { n + 1 } ) \delta _ { x _ { 2 } } \left(\left(C^{n+1} \phi\right)^{-1}\right.\right. \\
& \left.\left.\left.\left(1+\frac{h}{2}\left|\tilde{U}_{3}^{n+1}\right| D_{\alpha}^{-1}\left(C^{n+1}\right)\right)^{-1} \delta_{\bar{x}_{3}}\left(D_{\alpha}\left(C^{n+1}\right) \delta_{x_{3}} d_{t} S_{\alpha}^{n}\right)\right)\right)_{i j k}\right\}+(\Delta t)^{3}\left(1+\frac{h}{2}\left|\tilde{U}_{1}^{n+1}\right| D_{\alpha}^{-1}\left(C^{n+1}\right)\right)_{i j k}^{-1} \\
& \delta_{\bar{x}_{1}}\left(D _ { \alpha } ( C ^ { n + 1 } ) \delta _ { x _ { 1 } } \left(( C ^ { n + 1 } \phi ) ^ { - 1 } ( 1 + \frac { h } { 2 } | \tilde { U } _ { 2 } ^ { n + 1 } | D _ { \alpha } ^ { - 1 } ( C ^ { n + 1 } ) ) ^ { - 1 } \delta _ { \overline { x } _ { 2 } } \left(D _ { \alpha } ( C ^ { n + 1 } ) \delta _ { x _ { 2 } } \left(\left(C^{n+1} \phi\right)^{-1}(1+\right.\right.\right.\right. \\
& \left.\left.\left.\left.\left.+\frac{h}{2}\left|\tilde{U}_{3}^{n+1}\right| D_{\alpha}^{-1}\left(C^{n+1}\right)\right)^{-1} \delta_{\bar{x}_{3}}\left(D_{\alpha}\left(C^{n+1}\right) \delta_{x_{3}} d_{t} S_{\alpha}^{n}\right)\right)\right)\right)\right)_{i j k} \\
& +\Delta t\left\{\left(1+\frac{h}{2}\left|\tilde{U}_{1}^{n+1}\right| D_{\alpha}^{-1}\left(C^{n+1}\right)\right)_{i j k}^{-1} \delta_{\bar{x}_{1}}\left(D_{\alpha}\left(C^{n+1}\right) \delta_{x_{1}}\left(\left(C^{n+1} \phi\right)^{-1} \sum_{\beta=1}^{3} \delta_{\tilde{U}_{\beta}^{n+1}, x_{\beta}} S_{\alpha}^{n+1}\right)\right)_{i j k}\right. \\
& \left.+\left(1+\frac{h}{2}\left|\tilde{U}_{2}^{n+1}\right| D_{\alpha}^{-1}\left(C^{n+1}\right)\right)_{i j k}^{-1} \delta_{\bar{x}_{2}}\left(D_{\alpha}\left(C^{n+1}\right) \delta_{x_{2}}\left(\left(C^{n+1} \phi\right)^{-1} \sum_{\beta=1}^{3} \delta_{\tilde{U}_{\beta}^{n+1}, x_{\beta}} S_{\alpha}^{n+1}\right)\right)_{i j k}\right\} \\
& -(\Delta t)^{2}\left(1+\frac{h}{2}\left|\tilde{U}_{1}^{n+1}\right| D_{\alpha}^{-1}\left(C^{n+1}\right)\right)_{i j k}^{-1} \delta_{\bar{x}_{1}}\left(D _ { \alpha } ( C ^ { n + 1 } ) \delta _ { x _ { 1 } } \left(\left(C^{n+1} \phi\right)^{-1}\left(1+\frac{h}{2}\left|\tilde{U}_{2}^{n+1}\right| D_{\alpha}^{-1}\left(C^{n+1}\right)\right)^{-1}\right.\right. \\
& \left.\left.\delta_{\bar{x}_{2}}\left(D_{\alpha}\left(C^{n+1}\right) \delta_{x_{2}}\left(\left(C^{n+1} \phi\right)^{-1} \sum_{\beta=1}^{3} \delta_{\tilde{U}_{\beta}^{n+1}, x_{\beta}} S_{\alpha}^{n+1}\right)\right)\right)\right)_{i j k}, 1 \leq i, j, k \leq N-1, \alpha=1,2, \cdots, n_{c} \text {, } \\
& S_{\alpha, i j k}^{n+1}=h_{\alpha, i j k}^{n+1}, X_{i j k} \in \partial \Omega_{h}, \alpha=1,2, \cdots, n_{c} .
\end{aligned}
$$

Error equation of components concentration is derived by (7) $\left(t=t^{n+1}\right)$ and (48),

$$
\begin{aligned}
& \phi_{i j k} C_{i j k}^{n+1} \frac{\zeta_{\alpha, i j k}^{n+1}-\zeta_{\alpha, i j k}^{n}}{\Delta t}-\sum_{\beta=1}^{3}\left(1+\frac{h}{2}\left|\tilde{U}_{\beta}^{n+1}\right| D_{\alpha}^{-1}\left(C^{n+1}\right)\right)_{i j k}^{-1} \delta_{\bar{x}_{\beta}}\left(D_{\alpha}\left(C^{n+1}\right) \delta_{x_{\beta}} \zeta_{\alpha}^{n+1}\right)_{i j k} \\
& =\left\{\phi\left(C^{n+1}-c^{n+1}\right) \frac{\partial s_{\alpha}}{\partial t}\right\}_{i j k}+\sum_{\beta=1}^{3}\left(1+\frac{h}{2}\left|\tilde{U}_{\beta}^{n+1}\right| D_{\alpha}^{-1}\left(C^{n+1}\right)\right)_{i j k}^{-1} \delta_{\bar{x}_{\beta}}\left(\left[D_{\alpha}\left(c^{n+1}\right)-D_{\alpha}\left(C^{n+1}\right)\right] \delta_{x_{\beta}} S_{\alpha}^{n+1}\right)_{i j k}
\end{aligned}
$$




$$
\begin{aligned}
& +\sum_{\beta=1}^{3}\left[\left(1+\frac{h}{2}\left|u_{\beta}^{n+1}\right| D_{\alpha}^{-1}\left(c^{n+1}\right)\right)^{-1}-\left(1+\frac{h}{2}\left|\tilde{U}_{\beta}^{n+1}\right| D_{\alpha}^{-1}\left(C^{n+1}\right)\right)^{-1}\right]_{i j k} \delta_{\bar{x}_{\beta}}\left(D_{\alpha}\left(c^{n+1}\right) \delta_{x_{\beta}} S_{\alpha}^{n+1}\right)_{i j k} \\
& +\sum_{\beta=1}^{3}\left\{\delta_{\bar{U}_{\beta}^{n+1}, x_{\beta}} S_{\alpha}^{n+1}-\delta_{u_{\beta}^{n+1}, x_{\beta}} s_{\alpha}^{n+1}\right\}_{i j k}+Q_{\alpha}\left(c_{i j k}^{n+1}, s_{\alpha, i j k}^{n+1}\right)-Q_{\alpha}\left(C_{i j k}^{n+1}, S_{\alpha, i j k}^{n+1}\right) \\
& +\left\{\left(S_{\alpha}^{n} q\left(C^{n+1}\right)-s_{\alpha}^{n} q\left(c^{n+1}\right)\right)_{i j k}+\left(s_{\alpha}^{n+1} d\left(c^{n+1}\right) \frac{\partial p^{n+1}}{\partial t}-S_{\alpha}^{n} d\left(C^{n+1}\right) \frac{P^{n+1}-P^{n}}{\Delta t}\right)_{i j k}\right. \\
& \left.+\left(S_{\alpha}^{n} \phi \frac{C^{n+1}-C^{n}}{\Delta t}-s_{\alpha}^{n+1} \phi \frac{\partial c^{n+1}}{\partial t}\right)_{i j k}\right\}-(\Delta t)^{2}\left\{( 1 + \frac { h } { 2 } | \tilde { U } _ { 1 } ^ { n + 1 } | D _ { \alpha } ^ { - 1 } ( C ^ { n + 1 } ) ) _ { i j k } ^ { - 1 } \delta _ { \overline { x } _ { 1 } } \left(D_{\alpha}\left(C^{n+1}\right) \delta_{x_{1}}(\right.\right. \\
& \left.\left.\left(C^{n+1} \phi\right)^{-1}\left(1+\frac{h}{2}\left|\tilde{U}_{2}^{n+1}\right| D_{\alpha}^{-1}\left(C^{n+1}\right)\right)^{-1} \delta_{\bar{x}_{2}}\left(D_{\alpha}\left(C^{n+1}\right) \delta_{x_{2}} d_{t} \zeta_{\alpha}^{n}\right)\right)\right)_{i j k}+\cdots+\left[\left(1+\frac{h}{2}\left|u_{1}^{n+1}\right| D_{\alpha}^{-1}\left(c^{n+1}\right)\right)^{-1}\right. \\
& \left.-\left(1+\frac{h}{2}\left|\tilde{U}_{1}^{n+1}\right| D_{\alpha}^{-1}\left(C^{n+1}\right)\right)^{-1}\right]_{i j k} \delta_{\bar{x}_{1}}\left(D _ { \alpha } ( c ^ { n + 1 } ) \delta _ { x _ { 1 } } \left(( c ^ { n + 1 } \phi ) ^ { - 1 } ( 1 + \frac { h } { 2 } | u _ { 2 } ^ { n + 1 } | D _ { \alpha } ^ { - 1 } ( c ^ { n + 1 } ) ) ^ { - 1 } \delta _ { \overline { x } _ { 2 } } \left(D_{\alpha}\left(c^{n+1}\right)\right.\right.\right. \\
& \left.\left.\left.\delta_{x_{2}} d_{t} s_{\alpha}^{n+1}\right)\right)\right)_{i j k}+\left(1+\frac{h}{2}\left|\tilde{U}_{1}^{n+1}\right| D_{\alpha}^{-1}\left(C^{n+1}\right)\right)_{i j k}^{-1} \delta_{\bar{x}_{1}}\left(D _ { \alpha } ( C ^ { n + 1 } ) \delta _ { x _ { 1 } } \left(\left(C^{n+1} \phi\right)^{-1}\left(1+\frac{h}{2}\left|\tilde{U}_{3}^{n+1}\right| D_{\alpha}^{-1}\left(C^{n+1}\right)\right)^{-1}\right.\right. \\
& \left.\left.\delta_{\bar{x}_{3}}\left(D_{\alpha}\left(C^{n+1}\right) \delta_{x_{3}} d_{t} \zeta_{\alpha}^{n}\right)\right)\right)_{i j k}+\cdots+\left(1+\frac{h}{2}\left|\tilde{U}_{2}^{n+1}\right| D_{\alpha}^{-1}\left(C^{n+1}\right)\right)_{i j k}^{-1} \delta_{\bar{x}_{2}}\left(D _ { \alpha } ( C ^ { n + 1 } ) \delta _ { x _ { 2 } } \left(\left(C^{n+1} \phi\right)^{-1}\right.\right. \\
& \left.\left.\left.\left(1+\frac{h}{2}\left|\tilde{U}_{3}^{n+1}\right| D_{\alpha}^{-1}\left(C^{n+1}\right)\right)^{-1} \delta_{\bar{x}_{3}}\left(D_{\alpha}\left(C^{n+1}\right) \delta_{x_{3}} d_{t} \zeta_{\alpha}^{n}\right)\right)\right)_{i j k}+\cdots\right\}+(\Delta t)^{3}\left\{\left(1+\frac{h}{2}\left|\tilde{U}_{1}^{n+1}\right| D_{\alpha}^{-1}\left(C^{n+1}\right)\right)_{i j k}^{-1}\right. \\
& \delta_{\bar{x}_{1}}\left(D _ { \alpha } ( C ^ { n + 1 } ) \delta _ { x _ { 1 } } \left(( C ^ { n + 1 } \phi ) ^ { - 1 } ( 1 + \frac { h } { 2 } | \tilde { U } _ { 2 } ^ { n + 1 } | D _ { \alpha } ^ { - 1 } ( C ^ { n + 1 } ) ) ^ { - 1 } \delta _ { \overline { x } _ { 2 } } \left(D _ { \alpha } ( C ^ { n + 1 } ) \delta _ { x _ { 2 } } \left(( C ^ { n + 1 } \phi ) ^ { - 1 } \left(1+\frac{h}{2}\left|\tilde{U}_{3}^{n+1}\right|\right.\right.\right.\right.\right. \\
& \left.\left.\left.\left.\left.\left.D_{\alpha}^{-1}\left(C^{n+1}\right)\right)^{-1} \delta_{\bar{x}_{3}}\left(D_{\alpha}\left(C^{n+1}\right) \delta_{x_{3}} d_{t} \zeta_{\alpha}^{n}\right)\right)\right)\right)\right)_{i j k}+\cdots\right\} \\
& +\Delta t\left\{\left(1+\frac{h}{2}\left|\tilde{U}_{1}^{n+1}\right| D_{\alpha}^{-1}\left(C^{n+1}\right)\right)_{i j k}^{-1} \delta_{\bar{x}_{1}}\left(D_{\alpha}\left(C^{n+1}\right) \delta_{x_{1}}\left(\left(C^{n+1} \phi\right)^{-1} \sum_{\beta=1}^{3} \delta_{\tilde{U}_{\beta}^{n+1}, x_{\beta}} \zeta_{\alpha}^{n+1}\right)\right)_{i j k}+\cdots\right. \\
& \left.+\left(1+\frac{h}{2}\left|\tilde{U}_{2}^{n+1}\right| D_{\alpha}^{-1}\left(C^{n+1}\right)\right)_{i j k}^{-1} \delta_{\bar{x}_{2}}\left(D_{\alpha}\left(C^{n+1}\right) \delta_{x_{2}}\left(\left(C^{n+1} \phi\right)^{-1} \sum_{\beta=1}^{3} \delta_{\tilde{U}_{\beta}^{n+1}, x_{\beta}} \zeta_{\alpha}^{n+1}\right)\right)_{i j k}+\cdots\right\} \\
& -(\Delta t)^{2}\left\{( 1 + \frac { h } { 2 } | \tilde { U } _ { 1 } ^ { n + 1 } | D _ { \alpha } ^ { - 1 } ( C ^ { n + 1 } ) ) _ { i j k } ^ { - 1 } \delta _ { \overline { x } _ { 1 } } \left(D _ { \alpha } ( C ^ { n + 1 } ) \delta _ { x _ { 1 } } \left(\left(C^{n+1} \phi\right)^{-1}\left(1+\frac{h}{2}\left|\tilde{U}_{2}^{n+1}\right| D_{\alpha}^{-1}\left(C^{n+1}\right)\right)^{-1}\right.\right.\right. \\
& \left.\left.\left.\delta_{\bar{x}_{2}}\left(D_{\alpha}\left(C^{n+1}\right) \delta_{x_{2}}\left(\left(C^{n+1} \phi\right)^{-1} \sum_{\beta=1}^{3} \delta_{\tilde{U}_{\beta}^{n+1}, x_{\beta}} \zeta_{\alpha}^{n+1}\right)\right)\right)\right)_{i j k}+\cdots\right\}+\varepsilon_{\alpha, i j k}, \\
& 1 \leq i, j, k \leq N-1, \alpha=1,2, \cdots, n_{c}, \\
& \zeta_{\alpha, i j k}^{n+1}=0, X_{i j k} \in \partial \Omega_{h}, \alpha=1,2, \cdots, n_{c},
\end{aligned}
$$

where $\left|\varepsilon_{\alpha, i j k}\right| \leq M\left\{h^{2}+\Delta t\right\}, \alpha=1,2, \cdots, n_{c}$.

In numerical analysis there exists bound water in oil reservoir, that is to say there exists a positive constant $c_{*}$ such that $c(X, t) \geq c_{*}>0$. It holds as $h$ and $\Delta t$ sufficiently small because of the convergence result of $c(X, t)(17 \mathrm{a})$,

$$
C(X, t) \geq \frac{c_{*}}{2} .
$$

Multiplying both sides of (49) by $\delta_{t} \zeta_{\alpha, i j k}^{n}=d_{t} \zeta_{\alpha, i j k}^{n} \Delta t=\zeta_{\alpha, i j k}^{n+1}-\zeta_{\alpha, i j k}^{n}$ and making inner product,

$$
\begin{aligned}
& \left\langle\phi C^{n+1} \frac{\zeta_{\alpha}^{n+1}-\zeta_{\alpha}^{n}}{\Delta t}, d_{t} \zeta_{\alpha}^{n}\right\rangle \Delta t+\sum_{\beta=1}^{3}\left\langle D_{\alpha}\left(C^{n+1}\right) \delta_{x_{\beta}} \zeta_{\alpha}^{n+1}, \delta_{x_{\beta}}\left[\left(1+\frac{h}{2}\left|\tilde{U}_{\beta}^{n+1}\right| D_{\alpha}^{-1}\left(C^{n+1}\right)\right)^{-1}\left(\zeta_{\alpha}^{n+1}-\zeta_{\alpha}^{n}\right)\right]\right\rangle \\
& =\left\langle\phi\left(C^{n+1}-c^{n+1}\right) \frac{\partial s_{\alpha}}{\partial t}, d_{t} \zeta_{\alpha}^{n}\right\rangle \Delta t \\
& +\sum_{\beta=1}^{3}\left\{\left\langle\left(1+\frac{h}{2}\left|\tilde{U}_{\beta}^{n+1}\right| D_{\alpha}^{-1}\left(C^{n+1}\right)\right)^{-1} \delta_{\bar{x}_{\beta}}\left(\left[D_{\alpha}\left(c^{n+1}\right)-D_{\alpha}\left(C^{n+1}\right)\right] \delta_{x_{\beta}} S_{\alpha}^{n+1}\right), d_{t} \zeta_{\alpha}^{n}\right\rangle\right. \\
& \left.+\left\langle\left[\left(1+\frac{h}{2}\left|u_{\beta}^{n+1}\right| D_{\alpha}^{-1}\left(c^{n+1}\right)\right)^{-1}-\left(1+\frac{h}{2}\left|\tilde{U}_{\beta}^{n+1}\right| D_{\alpha}^{-1}\left(C^{n+1}\right)\right)^{-1}\right] \delta_{\bar{x}_{\beta}}\left(D_{\alpha}\left(c^{n+1}\right) \delta_{x_{\beta}} \zeta_{\alpha}^{n+1}\right), d_{t} \zeta_{\alpha}^{n}\right\rangle\right\} \Delta t
\end{aligned}
$$




$$
\begin{aligned}
& +\sum_{\beta=1}^{3}\left\langle\delta_{U_{\beta}^{n+1}, x_{\beta}} S_{\alpha}^{n+1}-\delta_{u_{\beta}^{n+1}, x_{\beta}} s_{\alpha}^{n+1}, d_{t} \zeta_{\alpha}^{n}\right\rangle \Delta t+\left\langle Q_{\alpha}\left(c^{n+1}, s_{\alpha}^{n+1}\right)-Q_{\alpha}\left(C^{n+1}, S_{\alpha}^{n+1}\right), d_{t} \zeta_{\alpha}^{n}\right\rangle \Delta t \\
& +\left\langle S_{\alpha}^{n} q\left(C^{n+1}\right)-s_{\alpha}^{n} q\left(c^{n+1}\right), d_{t} \zeta_{\alpha}^{n}\right\rangle \Delta t+\left\langle s_{\alpha}^{n+1} d\left(c^{n+1}\right) \frac{\partial p^{n+1}}{\partial t}-S_{\alpha}^{n} d\left(C^{n+1}\right) \frac{P^{n+1}-P^{n}}{\Delta t}, d_{t} \zeta_{\alpha}^{n}\right\rangle \Delta t \\
& +\left\langle S_{\alpha}^{n} \phi \frac{C^{n+1}-C^{n}}{\Delta t}-s_{\alpha}^{n+1} \phi \frac{\partial c^{n+1}}{\partial t}, d_{t} \zeta_{\alpha}^{n}\right\rangle \Delta t \\
& -(\Delta t)^{3}\left\{\left\langle( 1 + \frac { h } { 2 } | \tilde { U } _ { 1 } ^ { n + 1 } | D _ { \alpha } ^ { - 1 } ( C ^ { n + 1 } ) ) ^ { - 1 } \delta _ { \overline { x } _ { 1 } } \left(D _ { \alpha } ( C ^ { n + 1 } ) \delta _ { x _ { 1 } } \left(\left(C^{n+1} \phi\right)^{-1}\left(1+\frac{h}{2}\left|\tilde{U}_{2}^{n+1}\right| D_{\alpha}^{-1}\left(C^{n+1}\right)\right)^{-1}\right.\right.\right.\right. \\
& \left.\left.\left.\delta_{\bar{x}_{2}}\left(D_{\alpha}\left(C^{n+1}\right) \delta_{x_{2}} d_{t} \zeta_{\alpha}^{n}\right)\right)\right), d_{t} \zeta^{n}\right\rangle+\cdots+\left\langle\left[\left(1+\frac{h}{2}\left|u_{1}^{n+1}\right| D_{\alpha}^{-1}\left(c^{n+1}\right)\right)^{-1}-\left(1+\frac{h}{2}\left|\tilde{U}_{1}^{n+1}\right| D_{\alpha}^{-1}\left(C^{n+1}\right)\right)^{-1}\right]\right. \\
& \left.\left.\delta_{\bar{x}_{1}}\left(D_{\alpha}\left(c^{n+1}\right) \delta_{x_{1}}\left(\left(c^{n+1} \phi\right)^{-1}\left(1+\frac{h}{2}\left|u_{2}^{n+1}\right| D_{\alpha}^{-1}\left(c^{n+1}\right)\right)^{-1} \delta_{\bar{x}_{2}}\left(D_{\alpha}\left(c^{n+1}\right) \delta_{x_{2}} d_{t} s_{\alpha}^{n+1}\right)\right)\right), d_{t} \zeta^{n}\right\rangle+\cdots\right\} \\
& +(\Delta t)^{4}\left\{\left\langle( 1 + \frac { h } { 2 } | \tilde { U } _ { 1 } ^ { n + 1 } | D _ { \alpha } ^ { - 1 } ( C ^ { n + 1 } ) ) ^ { - 1 } \delta _ { \overline { x } _ { 1 } } \left(D _ { \alpha } ( C ^ { n + 1 } ) \delta _ { x _ { 1 } } \left(\left(C^{n+1} \phi\right)^{-1}\left(1+\frac{h}{2}\left|\tilde{U}_{2}^{n+1}\right| D_{\alpha}^{-1}\left(C^{n+1}\right)\right)^{-1}\right.\right.\right.\right. \\
& \left.\left.\left.\left.\delta_{\bar{x}_{2}}\left(D_{\alpha}\left(C^{n+1}\right) \delta_{x_{2}}\left(\left(C^{n+1} \phi\right)^{-1}\left(1+\frac{h}{2}\left|\tilde{U}_{3}^{n+1}\right| D_{\alpha}^{-1}\left(C^{n+1}\right)\right)^{-1} \delta_{\bar{x}_{3}}\left(D_{\alpha}\left(C^{n+1}\right) \delta_{x_{3}} d_{t} \zeta_{\alpha}^{n}\right)\right)\right)\right)\right), d_{t} \zeta^{n}\right\rangle+\cdots\right\} \\
& +(\Delta t)^{2}\left\{\left\langle\left(1+\frac{h}{2}\left|\tilde{U}_{1}^{n+1}\right| D_{\alpha}^{-1}\left(C^{n+1}\right)\right)^{-1} \delta_{\bar{x}_{1}}\left(D_{\alpha}\left(C^{n+1}\right) \delta_{x_{1}}\left(\left(C^{n+1} \phi\right)^{-1} \sum_{\beta=1}^{3} \delta_{\tilde{U}_{\beta}^{n+1}, x_{\beta}} \zeta_{\alpha}^{n+1}\right)\right), d_{t} \zeta^{n}\right\rangle\right. \\
& +\cdots\}-(\Delta t)^{3}\left\{\left\langle( 1 + \frac { h } { 2 } | \tilde { U } _ { 1 } ^ { n + 1 } | D _ { \alpha } ^ { - 1 } ( C ^ { n + 1 } ) ) ^ { - 1 } \delta _ { \overline { x } _ { 1 } } \left(D _ { \alpha } ( C ^ { n + 1 } ) \delta _ { x _ { 1 } } \left(( C ^ { n + 1 } \phi ) ^ { - 1 } \left(1+\frac{h}{2}\left|\tilde{U}_{2}^{n+1}\right|\right.\right.\right.\right.\right. \\
& \left.\left.\left.\left.\left.D_{\alpha}^{-1}\left(C^{n+1}\right)\right)^{-1} \delta_{\bar{x}_{2}}\left(D_{\alpha}\left(C^{n+1}\right) \delta_{x_{2}}\left(\left(C^{n+1} \phi\right)^{-1} \sum_{\beta=1}^{3} \delta_{\tilde{U}_{\beta}^{n+1}, x_{\beta}} \zeta_{\alpha}^{n+1}\right)\right)\right)\right), d_{t} \zeta^{n}\right\rangle+\cdots\right\}+\left\langle\varepsilon_{\alpha}, d_{t} \zeta^{n}\right\rangle
\end{aligned}
$$

The left side terms of (51) are estimated first, by (17a),

$$
\begin{aligned}
& \left\langle\phi C^{n+1} d_{t} \zeta_{\alpha}^{n}, d_{t} \zeta_{\alpha}^{n}\right\rangle \Delta t \geq \frac{1}{2} \phi_{*} c_{*}\left\|d_{t} \zeta_{\alpha}^{n}\right\|^{2} \Delta t \\
& \left.\left.\sum_{\beta=1}^{3}\left\langle D_{\alpha}\left(C^{n+1}\right) \delta_{x_{\beta}} \zeta_{\alpha}^{n+1}, \delta_{x_{\beta}}\right|\left(1+\frac{h}{2}\left|\tilde{U}_{\beta}^{n+1}\right| D_{\alpha}^{-1}\left(C^{n+1}\right)\right)^{-1}\left(\zeta_{\alpha}^{n+1}-\zeta_{\alpha}^{n}\right)\right]\right\rangle \\
& \geq \frac{1}{2} \sum_{\beta=1}^{3}\left\{\left\langle D_{\alpha}\left(C^{n+1}\right) \delta_{x_{\beta}} \zeta_{\alpha}^{n+1},\left(1+\frac{h}{2}\left|\tilde{U}_{\beta}^{n+1}\right| D_{\alpha}^{-1}\left(C^{n+1}\right)\right)^{-1} \delta_{x_{\beta}} \zeta_{\alpha}^{n+1}\right\rangle-\left\langle D_{\alpha}\left(C^{n}\right) \delta_{x_{\beta}} \zeta_{\alpha}^{n},\left(1+\frac{h}{2}\left|\tilde{U}_{\beta}^{n}\right|\right.\right.\right. \\
& \left.\left.\left.D_{\alpha}^{-1}\left(C^{n}\right)\right)^{-1} \delta_{x_{\beta}} \zeta_{\alpha}^{n}\right\rangle\right\}-M \sum_{\beta=1}^{3}\left\|\delta_{x_{\beta}} \zeta_{\alpha}^{n+1}\right\|^{2} \Delta t-\varepsilon\left\|d_{t} \zeta_{\alpha}^{n}\right\|^{2} \Delta t .
\end{aligned}
$$

The right side terms of (51) are considered later.

$$
\begin{aligned}
& \left\langle\phi\left(C^{n+1}-c^{n+1}\right) \frac{\partial s_{\alpha}}{\partial t}, d_{t} \zeta_{\alpha}^{n}\right\rangle \Delta t \leq \varepsilon\left\|d_{t} \zeta_{\alpha}^{n}\right\|^{2} \Delta t+M\left\{h^{4}+(\Delta t)^{2}\right\} \Delta t, \\
& \sum_{\beta=1}^{3}\left\{\left\langle\left(1+\frac{h}{2}\left|\tilde{U}_{\beta}^{n+1}\right| D_{\alpha}^{-1}\left(C^{n+1}\right)\right)^{-1} \delta_{\bar{x}_{\beta}}\left(\left[D_{\alpha}\left(c^{n+1}\right)-D_{\alpha}\left(C^{n+1}\right)\right] \delta_{x_{\beta}} S_{\alpha}^{n+1}\right), d_{t} \zeta_{\alpha}^{n}\right\rangle\right. \\
& \left.+\left\langle\left[\left(1+\frac{h}{2}\left|u_{\beta}^{n+1}\right| D_{\alpha}^{-1}\left(c^{n+1}\right)\right)^{-1}-\left(1+\frac{h}{2}\left|\tilde{U}_{\beta}^{n+1}\right| D_{\alpha}^{-1}\left(C^{n+1}\right)\right)^{-1}\right] \delta_{\bar{x}_{\beta}}\left(D_{\alpha}\left(c^{n+1}\right) \delta_{x_{\beta}} \zeta_{\alpha}^{n+1}\right), d_{t} \zeta_{\alpha}^{n}\right\rangle\right\} \Delta t \\
& \leq \varepsilon\left\|d_{t} \zeta_{\alpha}^{n}\right\|^{2} \Delta t+M\left\{h^{4}+(\Delta t)^{2}\right\} \Delta t, \\
& \sum_{\beta=1}^{3}\left\langle\delta_{\bar{U}_{\beta}^{n+1}, x_{\beta}} S_{\alpha}^{n+1}-\delta_{u_{\beta}^{n+1}, x_{\beta}} s_{\alpha}^{n+1}, d_{t} \zeta_{\alpha}^{n}\right\rangle \Delta t \leq \varepsilon\left\|d_{t} \zeta_{\alpha}^{n}\right\|^{2} \Delta t+M\left\{\left\|\mathbf{u}^{n+1}-\mathbf{U}^{n+1}\right\|^{2}+\left\|\nabla_{h} \zeta_{\alpha}^{n+1}\right\|^{2}\right\} \Delta t \\
& \leq \varepsilon\left\|d_{t} \zeta_{\alpha}^{n}\right\|^{2} \Delta t+M\left\{\left\|\nabla_{h} \zeta_{\alpha}^{n+1}\right\|^{2}+h^{4}+(\Delta t)^{2}\right\} \Delta t, \\
& \left\langle Q_{\alpha}\left(c^{n+1}, s_{\alpha}^{n+1}\right)-Q_{\alpha}\left(C^{n+1}, S_{\alpha}^{n+1}\right), d_{t} \zeta_{\alpha}^{n}\right\rangle \Delta t \leq \varepsilon\left\|d_{t} \zeta_{\alpha}^{n}\right\|^{2} \Delta t+M\left\{\left\|\zeta_{\alpha}^{n+1}\right\|^{2}+h^{4}+(\Delta t)^{2}\right\} \Delta t, \\
& \left\langle S_{\alpha}^{n} q\left(C^{n+1}\right)-s_{\alpha}^{n} q\left(c^{n+1}\right), d_{t} \zeta_{\alpha}^{n}\right\rangle \Delta t \leq \varepsilon\left\|d_{t} \zeta_{\alpha}^{n}\right\|^{2} \Delta t+M\left\{\left\|\zeta_{\alpha}^{n}\right\|^{2}+h^{4}+(\Delta t)^{2}\right\} \Delta t, \\
& \left\langle s_{\alpha}^{n+1} d\left(c^{n+1}\right) \frac{\partial p^{n+1}}{\partial t}-S_{\alpha}^{n} d\left(C^{n+1}\right) \frac{P^{n+1}-P^{n}}{\Delta t}, d_{t} \zeta_{\alpha}^{n}\right\rangle \Delta t \leq \varepsilon\left\|d_{t} \zeta_{\alpha}^{n}\right\|^{2} \Delta t+M\left\{\left\|\zeta_{\alpha}^{n}\right\|^{2}+h^{4}+(\Delta t)^{2}\right\} \Delta t,
\end{aligned}
$$




$$
\left\langle S_{\alpha}^{n} \phi \frac{C^{n+1}-C^{n}}{\Delta t}-s_{\alpha}^{n+1} \phi \frac{\partial c^{n+1}}{\partial t}, d_{t} \zeta_{\alpha}^{n}\right\rangle \Delta t \leq \varepsilon\left\|d_{t} \zeta_{\alpha}^{n}\right\|^{2} \Delta t+M\left\{\left\|\zeta_{\alpha}^{n}\right\|^{2}+h^{4}+(\Delta t)^{2}\right\} \Delta t .
$$

For the last five terms of the right side of (51),

$$
\begin{aligned}
& (\Delta t)^{3}\left\{\left\langle( 1 + \frac { h } { 2 } | \tilde { U } _ { 1 } ^ { n + 1 } | D _ { \alpha } ^ { - 1 } ( C ^ { n + 1 } ) ) ^ { - 1 } \delta _ { \overline { x } _ { 1 } } \left(D _ { \alpha } ( C ^ { n + 1 } ) \delta _ { x _ { 1 } } \left(\left(C^{n+1} \phi\right)^{-1}\left(1+\frac{h}{2}\left|\tilde{U}_{2}^{n+1}\right| D_{\alpha}^{-1}\left(C^{n+1}\right)\right)^{-1}\right.\right.\right.\right. \\
& \left.\left.\left.\left.\delta_{\bar{x}_{2}}\left(D_{\alpha}\left(C^{n+1}\right) \delta_{x_{2}} d_{t} \zeta_{\alpha}^{n}\right)\right)\right), d_{t} \zeta^{n}\right\rangle+\cdots\right\}+\cdots+\left\langle\varepsilon_{\alpha}, d_{t} \zeta_{\alpha}^{n}\right\rangle \Delta t \\
& \leq \varepsilon\left\|d_{t} \zeta_{\alpha}^{n}\right\|^{2} \Delta t+M\left\{\left\|\nabla_{h} \zeta_{\alpha}^{n+1}\right\|^{2}+h^{4}+(\Delta t)^{2}\right\} \Delta t .
\end{aligned}
$$

Applying (52) (54) on both sides of (51),

$$
\begin{gathered}
\frac{1}{2} \phi_{*} c_{*}\left\|d_{t} \zeta_{\alpha}^{n}\right\|^{2} \Delta t+\frac{1}{2} \sum_{\beta=1}^{3}\left\{\left\langle D_{\alpha}\left(C^{n+1}\right) \delta_{x_{\beta}} \zeta_{\alpha}^{n+1},\left(1+\frac{h}{2}\left|\tilde{U}_{\beta}^{n+1}\right| D_{\alpha}^{-1}\left(C^{n+1}\right)\right)^{-1} \delta_{x_{\beta}} \zeta_{\alpha}^{n+1}\right\rangle\right. \\
\left.-\left\langle D_{\alpha}\left(C^{n}\right) \delta_{x_{\beta}} \zeta_{\alpha}^{n},\left(1+\frac{h}{2}\left|\tilde{U}_{\beta}^{n}\right| D_{\alpha}^{-1}\left(C^{n}\right)\right)^{-1} \delta_{x_{\beta}} \zeta_{\alpha}^{n}\right\rangle\right\} \\
\leq \varepsilon\left\|d_{t} \zeta_{\alpha}^{n}\right\|^{2} \Delta t+M\left\{\left\|\nabla_{h} \zeta_{\alpha}^{n+1}\right\|^{2}+\left\|\zeta_{\alpha}^{n+1}\right\|^{2}+h^{4}+(\Delta t)^{2}\right\} \Delta t .
\end{gathered}
$$

Summing (55) on $t$ for $0 \leq n \leq L$, and noting that $\zeta_{\alpha}^{0}=0$,

$$
\begin{gathered}
\sum_{n=0}^{L}\left\|d_{t} \zeta_{\alpha}^{n}\right\|^{2} \Delta t+\sum_{\beta=1}^{3}\left\{\left\langle D_{\alpha}\left(C^{L+1}\right) \delta_{x_{\beta}} \zeta_{\alpha}^{L+1},\left(1+\frac{h}{2}\left|\tilde{U}_{\beta}^{L+1}\right| D_{\alpha}^{-1}\left(C^{L+1}\right)\right)^{-1} \delta_{x_{\beta}} \zeta_{\alpha}^{L+1}\right\rangle\right. \\
\left.-\left\langle D_{\alpha}\left(C^{0}\right) \delta_{x_{\beta}} \zeta_{\alpha}^{0},\left(1+\frac{h}{2}\left|\tilde{U}_{\beta}^{0}\right| D_{\alpha}^{-1}\left(C^{0}\right)\right)^{-1} \delta_{x_{\beta}} \zeta_{\alpha}^{0}\right\rangle\right\} \\
\leq M \sum_{n=0}^{L}\left\{\left\|\nabla_{h} \zeta_{\alpha}^{n+1}\right\|^{2}+\left\|\zeta_{\alpha}^{n+1}\right\|^{2}+h^{4}+(\Delta t)^{2}\right\} \Delta t
\end{gathered}
$$

Then,

$$
\sum_{n=0}^{L}\left\|d_{t} \zeta_{\alpha}^{n}\right\|^{2} \Delta t+\sum_{\beta=1}^{3} \mid\left[\delta_{x_{\beta}} \zeta_{\alpha}^{n+1} \|^{2} \leq M \sum_{n=0}^{L}\left\{\left\|\nabla_{h} \zeta_{\alpha}^{n+1}\right\|^{2}+\left\|\zeta_{\alpha}^{n+1}\right\|^{2}+h^{4}+(\Delta t)^{2}\right\} \Delta t\right.
$$

Noting that $\zeta_{\alpha}^{0}=0$,

$$
\left\|\zeta_{\alpha}^{L+1}\right\|^{2}=\varepsilon \sum_{n=0}^{L}\left\|d_{t} \zeta_{\alpha}^{n}\right\|^{2} \Delta t+M \sum_{n=0}^{L}\left\|\zeta_{\alpha}^{n}\right\|^{2} \Delta t
$$

continue,

$$
\sum_{n=0}^{L}\left\|d_{t} \zeta_{\alpha}^{n}\right\|^{2} \Delta t+\left\|\zeta_{\alpha}^{L+1}\right\|_{1}^{2} \leq M \sum_{n=0}^{L}\left\{\left\|\zeta_{\alpha}^{n+1}\right\|_{1}^{2}+h^{4}+(\Delta t)^{2}\right\} \Delta t
$$

Using the Gronwall lemma,

$$
\sum_{n=0}^{L}\left\|d_{t} \zeta_{\alpha}^{n}\right\|^{2} \Delta t+\left\|\zeta_{\alpha}^{L+1}\right\|_{1}^{2} \leq M\left\{h^{4}+(\Delta t)^{2}\right\}
$$

The proof ends.

\section{Fund}

This work is supported by the National Natural Science Foundation of China (Grant No: 11871312), Natural Science Foundation of Shandong Province (Grant No: ZR2016AM08)

\section{References}

Axelsson, O., \& Gustafasson, I. (1979). A modified upwind scheme for convective transport equations and the use of a conjugate gradient method for the solution of non-symmetric systems of equations. J. Inst. Maths. Applics., 23, 321-337. https://doi.org/10.1093/imamat/23.3.321 
Douglas, Jr. J., \& Gunn, J. E. (1963). Two order correct difference analogues for the equation of multidimensional heat flow. Math. Comp., 17(81), 71-80. https://doi.org/10.1090/S0025-5718-1963-0149676-2

Douglas, Jr. J., \& Gunn, J. E. (1964). A general formulation of alternating direction methods, Part 1. Parabolic and hyperbolic problems. Numer. Math., 6(5), 428-453. https://doi.org/10.1007/BF01386093

Douglas, Jr. J. (1981). Simulation of miscible displacement in porous media by a modified method of characteristic procedure, Lecture Notes in Mathematics, 912,Numerical Analysis, Proceedings, Dundee.

Douglas, Jr. J., \& Russell, T. F. (1982). Numerical method for convection-dominated diffusion problems based on combining the method of characteristics with finite element or finite difference procedures. SIAM J. Numer. Anal., 19(5), 871-885. https://doi.org/10.1137/0719063

Douglas, Jr. J. (1983). Finite difference methods for two-phase incompressible flow in porous media. SIAM J. Numer. Anal., 20(4), 681-696. https://doi.org/10.1137/0720046

Douglas, Jr. J., \& Roberts, J. E. (1983). Numerical method for a model for compressible miscible displacement in porous media. Math. Comp., 4(164), 441-459. https://doi.org/10.1090/S0025-5718-1983-0717695-3

Ewing, R. E. (1983). The mathematics of reservoir simulation. SIAM, Philadelphia. https://doi.org/10.1137/1.9781611971071

Ewing, R. E., Russell, T. F., \& Wheeler, M. F. (1984). Convergence analysis of an approximation of miscible displacement in porous media by mixed finite elements and a modified method of characteristics. Comp. Meth. Appl. Mech. Eng., 47(1-2), 73-92. https://doi.org/10.1016/0045-7825(84)90048-3

Ewing, R. E., Yuan, Y. R., \& Li, G. (1989). Finite element for chemical-flooding simulation. Proceeding of the 7th International conference finite element method in flow problems, 1264-1271. The University of Alabama in Huntsville, Huntsville, Alabama: UAHDRESS.

Ewing, R. E., Lazarov, R. D., \& Vassilevski, A. T. (1994). Finite difference scheme for parabolic problems on composite grids with refinement in time and space. SIAM J.Numer. Anal., 31(6), 1605-1622. https://doi.org/10.1137/0731083

Institute of Mathematics, Shandong University. (1995). Exploration Institute of Daqing Petroleum Administration. Research and application of the polymer flooding software (summary of "Eighth-Five" national key science and technology program, Grant No. 85-203-01-08).

Institute of Mathematics, Shandong University. (2006). Exploration and development Institute of Daqing Petroleum Limited Liability Corporation. Modification of solving mathematical models of the polymer and improvement of reservoir description.

Institute of Mathematics, Shandong University. (2011). Shengli Oilfield Branch, China Petroleum E Chemical Corporation. Research on key technology of high temperature and high salinity chemical agent displacement, Chapter 4 , $\S 4.1$ Numerical method, 83-106.

Lazarov, R. D., Mishev I. D., \& Vassilevski, P. S. (1996). Finite volume method for convection-diffusion problems. SIAM J. Numer. Anal., 33(1), 31-55. https://doi.org/10.1137/0733003

Marchuk, G. I. (1990). Splitting and alternating direction method, In: Ciarlet, P.G., Lions, J.L., eds. Handbook of Numerical Analysis. Paris: Elsevier Science Publishers BV, 197-460.

Peaceman, D. W. (1980). Fundamental of numerical reservoir simulation. Amsterdam: Elsevier.

Yuan, Y. R. (1992). Time stepping along characteristics for the finite element approximation of compressible miscible displacement in porous media. Math. Numer. Sinica, 14(4), 386-406.

Yuan, Y. R. (1993). Finite difference methods for a compressible miscible displacement problem in porous media. Math. Numer. Sinica, 15(1), 16-28.

Yuan, Y. R. (1994). Characteristic finite difference methods for moving boundary value problem of numerical simulation of oil deposit. Science in China, 37(A)(12), 1442-1453.

Yuan, Y. R. (1996). The characteristic mixed-finite element method and analysis for three-dimensional moving boundary value problem.Science in China, 39(A)(3), 276-288.

Yuan, Y. R. (1996). Finite difference method and analysis for three-dimensional semiconductor device of heat conduction. Science in China, 39(A)(11), 1140-1151.

Yuan, Y. R., Yang, D. P., \& Qi, L. Q. et al. (1998). Research on algorithms of applied software of the polymer. Qinlin 
Gang (editor in chief), Proceedings on chemical flooding, 246-253, Beijing: Petroleum Industry Press.

Yuan, Y. R. (2010). Theory and application of upwind finite difference method for moving boundary value problem of three-dimensional percolation coupled system. Science in China, 4O(A)(2), 103-126.

Yuan, Y. R. (2012). The second-order upwind finite difference fractional steps method for moving boundary value problem of nonlinear percolation coupled system. Science in China, 42(A)(8), 84-864.

Yuan, Y. R. (2013). Theory and application of numerical simulation of energy sources, basis of numerical simulation of chemical production (tertiary oil recovery), Chapter 3, 257-304. Beijing: Science Press.

Yuan, Y. R., Cheng, A. J., Yang, D. P., \& Li, C. F. (2014). Theory and application of numerical simulation of chemical flooding in high temperature and high halt reservoirs. International Journal of Geosciences, 5, 956-970. https://doi.org/10.4236/ijg.2014.59082

Yuan, Y, R., Cheng, A. J., Yang, D. P. \& Li, C. F. (2014). Theory and application of numerical simulation of permeation fluid mechanics of the polymer-black oil. Journal of Geography and Geology, 6(4), 12-28. https://doi.org/10.5539/jgg.v6n4p12

Yuan, Y, R., Cheng, A. J., Yang, D. P., \& Li, C. F. (2014). Numerical simulation of black oil-three compound combination flooding. International Journal of Chemistry, 6(4), 38-54. https://doi.org/10.5539/ijc.v6n4p38

\section{Copyrights}

Copyright for this article is retained by the author(s), with first publication rights granted to the journal.

This is an open-access article distributed under the terms and conditions of the Creative Commons Attribution license (http://creativecommons.org/licenses/by/4.0/). 